Chinese Journal of Organic Chemistry

\title{
亚砜脱氧还原方法研究进展
}

\author{
李伟林陈炫颖郑天骄邹祺* 陈文博* \\ ( ${ }^{a}$ 上海电力大学 上海市电力材料防护与新材料重点实验室 上海 200090)
}

\begin{abstract}
摘要 亚砜还原为硫醚作为有机合成中一类重要的转化, 其应用一直受到人们的广泛关注. 随着金属有机的发展, 研 究人员发展了许多条件温和、实用有效的亚砜还原方法. 从还原剂的种类出发, 详细介绍了近二十年里亚砜脱氧还原 反应的研究进展，分析了这些还原体系所具有的优势及其潜在不足，并探讨了部分反应机理，最后对亚砜还原领域的 发展进行了展望.

关键词 亚砜; 脱氧还原; 硫醚; 研究进展
\end{abstract}

\section{Research Progress on Reduction of Sulfoxides to Thiothers}

\author{
Li, Weilin Chen, Xuanying Zheng, Tianjiao Zou, Qi* Chen, Wenbo* \\ ( ${ }^{a}$ Shanghai Key Laboratory of Materials Protection and Advanced Materials in Electric Power, \\ Shanghai University of Electric Power, Shanghai 200090)
}

\begin{abstract}
As one of the most important transformations in organic synthesis, deoxygenation reduction of sulfoxides to thioethers has attracted wide attention in researches and applications. With the development of organicmetallic chemistry, many mild and practical methods have been developed to reduce sulfoxides. In this review, based on the types of the reducing agents, the research progress on the deoxygenation reduction of sulfoxides to thiothers in recent 20 years is summarized. In addition, the advantages and disadvantages of these reduction systems, some reaction mechanisms and the prospects of research in this field are discussed.
\end{abstract}

Keywords sulfoxide; deoxygenation reduction; thioether; research progress

亚砜类化合物作为含有亚硫酰基官能团的一类重 要中间体, 在医药等行业中已经得到了广泛的应用 ${ }^{[1]}$. 而通过亚砜还原得到的硫醚化合物则是各种商业药物 (如抗生素)和化学品(如燃油添加剂)中至关重要的组成 部分, 其在有机合成中占据着非常重要的地位 ${ }^{[2]}$. 亚砜 的脱氧还原反应作为一类重要转化, 在有机合成(特别 是不对称合成反应)中具有相当大的实用性, 比如在不 对称合成反应中, 经过立体选择性诱导, 可以通过将亚 砜还原为硫醚，从而除去手性的亚磺酰基.

19 世纪末, Michaelis 等 ${ }^{[3,4]}$ 对亚砜的还原反应做了 一些开创性的工作, 从那时起科研工作者便对发展高效 实用的亚砜还原体系产生了浓厚的兴趣, 各种有效的 还原体系如雨后春笋般进入了人们的视野. 1988 年, Madesclaire 等 ${ }^{[5]}$ 比较系统地介绍了早期的亚砜还原方法
以及各类亚砜还原体系的机理, 在那之后亚砜还原研究 领域得到了突飞猛进的发展 ${ }^{[6-10]}$, 同时也吸引了更多的 研究人员投身于该领域.

早期的亚砜还原体系往往会面临副反应、低产率、 后处理困难或反应条件苛刻等限制. 随着金属有机的发 展, 很多研究人员都选择在亚砜还原体系中加入各类金 属及其配合物或路易斯酸等作为催化剂, 以加速还原反 应的进行，更多操作简单、条件温和并且实用有效的亚 砜还原体系被研究人员所发掘. 本综述从还原剂的种类 出发, 详细介绍了近二十年来新发展的各类亚砜还原体 系, 客观分析了这些还原体系所具有的优势以及其潜在 的不足, 并对部分还原体系的机理进行了探讨. 通过对 这些亚砜还原方法的总结, 对亚砜还原领域的发展进行 了展望.

\footnotetext{
* Corresponding authors. E-mail: qzou@shiep.edu.cn; wenbochen@shiep.edu.cn Received January 18, 2019; revised March 30, 2019; published online April 16, 2019. Project supported by the Natural Science Foundation of Shanghai City (No. 17ZR1447100) and the Science and Technology Commission of Shanghai Municipality (No. 14DZ2261000).

上海市自然科学基金(No.17ZR1447100)和上海市科学技术委员会(No. 14DZ2261000)资助项目.
} 


\section{1 含磷化合物还原亚砜}

利用含磷化合物还原亚砜是比较常见的一种方法. 早在 1891 年, Michaelis 和 Godchaux ${ }^{[3]}$ 就发现五氯化磷 和二苯基亚砜可以反应生成 4-氯苯基甲基硫醚，但该方 法的不足之处在于还原过程中可能会发生氯化反应. 此 外, 在 1-吗啉基-1-环已烯存在的情况下, 各类亚砜可以 与五氯化磷反应生成相应的硫醚以及 2 -氯环己酮 ${ }^{[11]}$. Michaelis 等在早期亚砜还原领域开拓性的工作, 吸引 了越来越多的研究人员对亚砜还原反应进行研究.

2003 年, Faucher 等 ${ }^{[12]}$ 以三(2-羧基乙基)膦(TCEP)作 为还原剂, 1,4-二氧六环与水作为混合溶剂, 在回流的 条件下对一系列亚砜进行了还原. 在此之前研究人员经 常利用三烷基膦等含磷化合物对亚砜进行还原, 但后处 理时通常需要经过色谱分离操作以除去其中的副产物. 三羧基乙基膦结构中的羧基可以大大提高其自身与相 应氧化物的水溶性, 因此以其作为还原剂, 在反应结束 之后可以直接萃取分离得到所需的最终产物. 在还原一 些反应活性较低的亚砜或带有不能经受长时间加热反 应性官能团的亚砜时，可向反应体系中加入一定量的 $\mathrm{I}_{2}$ 来催化反应的进行(Eq. 1).

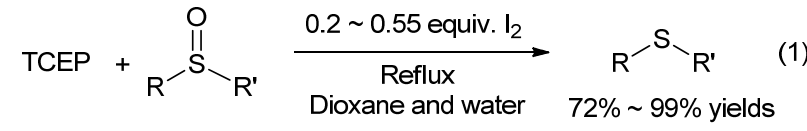

$\mathrm{R}, \mathrm{R}^{\prime}$ = phenyl, benzyl, alkyl<smiles>c1ccc(Sc2ccccc2)cc1</smiles>

$86 \%$ yield

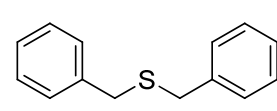

$99 \%$ yield

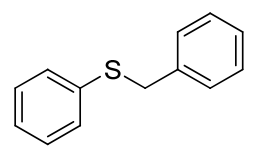

$97 \%$ yield
2004 年, $\mathrm{Sanz}$ 等 ${ }^{[13]}$ 利用 $\mathrm{MoO}_{2} \mathrm{Cl}_{2}(\mathrm{dmf})_{2}$ 作为催化剂, $\mathrm{P}(\mathrm{OPh})_{3}$ 作为还原剂, 在乙腈中对一系列亚砜进行了还 原(Eq. 2). 亚磷酸酯比三芳基膦具有更高的反应性, 比 较容易通过氧化的形式除去. 其缺点在于, 大多数情况 下反应都需要相对较高的温度以及较长的反应时间. 因 此在体系中加入一定量的 $\mathrm{MoO}_{2} \mathrm{Cl}_{2}(\mathrm{dmf})_{2}$ 作为催化剂来 催化反应的进行, 最终得到亚砜收率较高且化学选择性 良好.

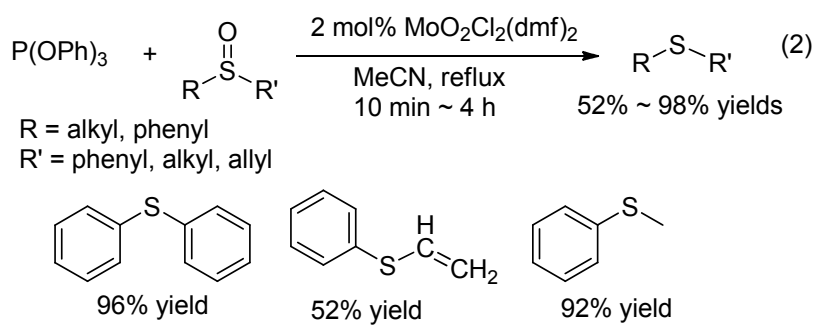

随后 2005 年 Kikuchi 等 ${ }^{[14]}$ 报道了 $\mathrm{PPh}_{3} / \mathrm{TiCl}_{4}$ 的亚砜
还原体系. $\mathrm{PPh}_{3}$ 是一种相对常见的用于还原亚砜的还原 剂, 然而, $\mathrm{PPh}_{3}$ 还原亚砜时反应条件十分剧烈，且底物 的适用范围有限. 因此受三苯基膦/路易斯酸体系对 $\alpha$ 溴代羧酸衍生物等还原的启发后，希望使用类似的还原 体系对亚砜进行还原. 在进行条件优化之后, 确定 $\mathrm{TiCl}_{4}$ 为最适合该体系的路易斯酸. 该方法的反应条件温和, 操作方法简便, 底物的普适性较好(Eq. 3).

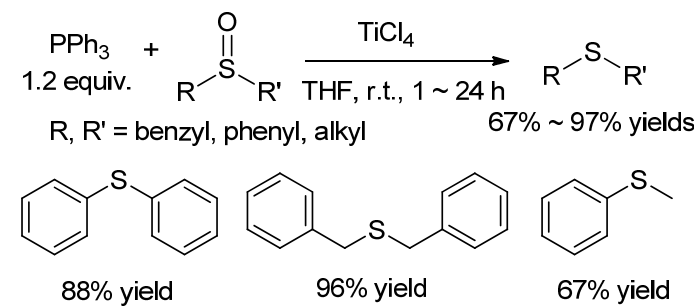

同年，Iranpoor 等 ${ }^{[15]}$ 发现非均相膦试剂 Silphos $\left[\mathrm{PCl}_{3-n}\left(\mathrm{SiO}_{2}\right)_{n}\right]$ 和 $\mathrm{I}_{2}$ 组成的体系可以在干燥的乙腈中以 高收率还原一系列亚砜(Eq. 4). 与其他使用膦试剂的文 献方法相比, $\left[\mathrm{PCl}_{3-n}\left(\mathrm{SiO}_{2}\right)_{n}\right]$ 的优点在于，易制备(由硅胶 和 $\mathrm{PCl}_{3}$ 制备), 易加工, 所产生的氧化副产物可通过简 单的过滤除去等. 但值得注意的是，在没有分子碘催化 的情况下, Silphos 不能单独完成亚砜的还原.

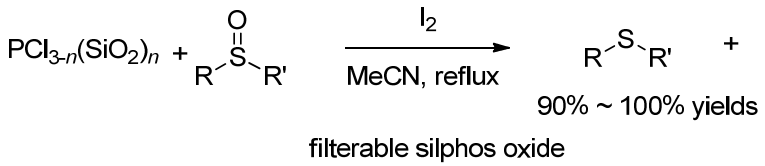

$\mathrm{R}, \mathrm{R}^{\prime}=$ phenyl, benzyl, alkyl, allyl

2007 年, Woollins 和 $\mathrm{Hua}^{[16]}$ 在利用 Woollins 试剂作 偶联反应过程中，发现该试剂可以用来还原二苯基亚 砜，且分离收率高达 99\%. 于是他们进一步利用该试剂 对其它的烷基亚砜和芳基亚砜进行了还原尝试(Eq. 5), 并成功还原了一系列烷基亚砜和芳基亚砜，且反应的条 件比较温和, 具有高选择性和高转化率. 不过, 作者继 续利用该反应试剂对砜类化合物进行还原时发现，反应 不能发生. 最后作者对该反应的机理进行了探究 (Scheme 1).

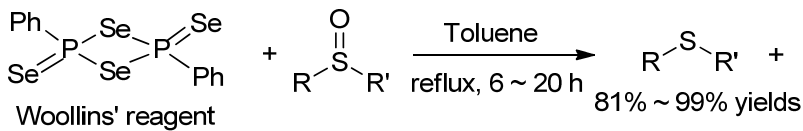

$$
\begin{aligned}
& \left(\mathrm{PhPO}_{2}\right)_{3}+\mathrm{Se} \\
& \mathrm{R}, \mathrm{R}^{\prime}=\text { phenyl, alkyl, benzyl }
\end{aligned}
$$

同年, Bahramiet 等 ${ }^{[17]}$ 利用 $\mathrm{PPh}_{3} / \mathrm{Br}_{2} / \mathrm{CuBr}$ 体系对各 类亚砜进行了还原，该反应在乙腈中回流，以相当高的 收率得到了亚砜还原后的产物(Eq. 6), 且体系具有较好 的化学选择性与官能团容忍性(如碳-碳双键和酮等等). 


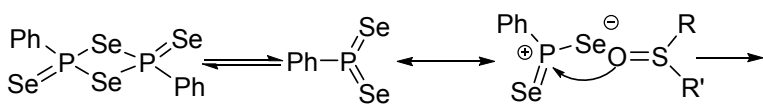

Woollins' reagent
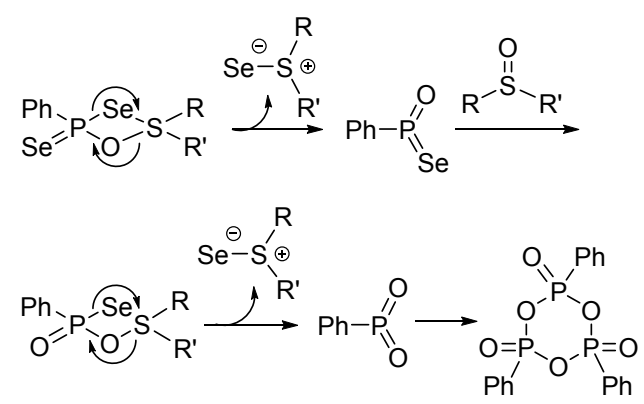

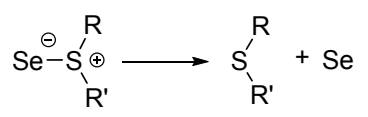

图式 1 Woollins 试剂还原亚砜的机理研究

Scheme 1 Proposed mechanism for the reduction of sulfoxides with Woollins' reagent

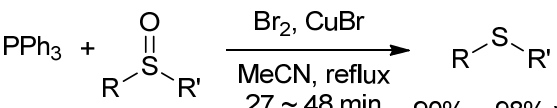

$$
\begin{aligned}
& \mathrm{R}=\text { aryl, alkyl } \quad 27 \sim 48 \mathrm{~min} \quad 90 \% \sim 98 \% \text { yields } \\
& \mathrm{R}^{\prime} \text { = alkyl, benzyl, aryl, allyl }
\end{aligned}
$$

随后作者利用二茶基亚砜作为模板底物对 $\mathrm{Ph}_{3} \mathrm{P}$ 、 $\mathrm{Ph}_{3} \mathrm{P} / \mathrm{Br}_{2} 、 \mathrm{Ph}_{3} \mathrm{P} / \mathrm{CuBr}$ 以及 $\mathrm{CuBr}$ 等体系进行了探究, 发 现在这些体系中亚砜的脱氧反应均不能发生. 最后对该 反应的机理进行了探讨: $\mathrm{Br}_{2}$ 与 $\mathrm{Ph}_{3} \mathrm{P}$ 反应形成中间体 $\mathbf{1 , 1}$ 与亚砜反应生成中间体 $\mathbf{2}$, 最后在 $\mathrm{CuBr}$ 存在下中间体 $\mathbf{2}$ 转化为相应的硫化物(Scheme 2).

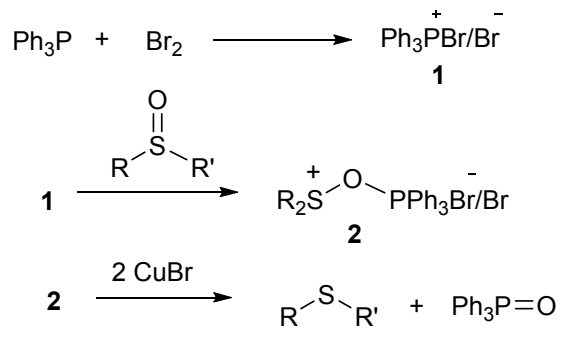

图式 $2 \mathrm{PPh}_{3} / \mathrm{Br}_{2} / \mathrm{CuBr}$ 体系还原亚砜的机理研究

Scheme 2 Proposed mechanism for sulfoxide reduction catalyzed by $\mathrm{PPh}_{3} / \mathrm{Br}_{2} / \mathrm{CuBr}$

2012 年, Bagherzadeh 和 Ghazali-Esfahani ${ }^{[18]}$ 利用 $[\mathrm{RMIM}]_{2}\left[\mathrm{Mo}(\mathrm{O})_{2}(\mathrm{NCS})_{4}\right]$ 作为催化剂, $\mathrm{PPh}_{3}$ 作为还原剂, 在 $\left[\mathrm{RMIm} \mathrm{PF}_{6}\right.$ 和甲苯的混合溶剂中, 以高收率将亚砜转 化为相应的硫醚(Eq. 7). 作者使用该混合溶剂的目的是 促进催化剂的再循环性, 以改善体系在催化剂/溶剂再 循环方面的问题, 并最终使得该体系即使经过 8 个循环 也不会出现催化剂活性的损失. 反应机理如 Scheme 3 所示.

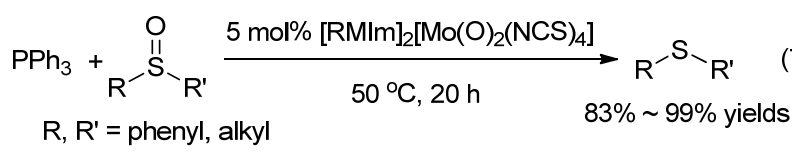

RMIM = 1-butyl-3-methylimidazolium

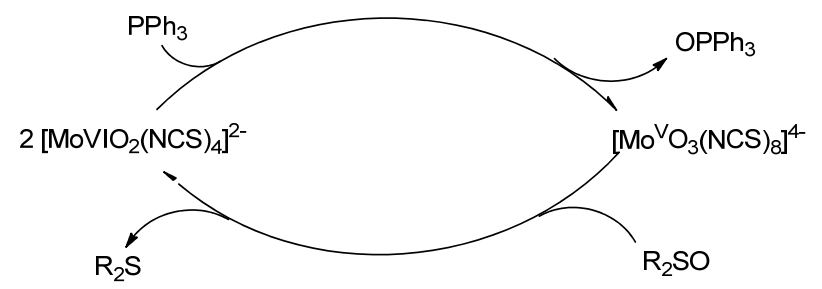

图式 $3[\mathrm{RMIm}]_{2}\left[\mathrm{Mo}(\mathrm{O})_{2}(\mathrm{NCS})_{4}\right]$ 催化的亚砜还原机理 Scheme 3 Proposed mechanism for sulfoxide reduction with $[\mathrm{RMIm}]_{2}\left[\mathrm{Mo}(\mathrm{O})_{2}(\mathrm{NCS})_{4}\right]$

2013 年, Jang 等 ${ }^{[19]}$ 报道了在四氢呋喃中以 $\mathrm{PPh}_{3}$ 作为 还原剂, $\mathrm{SOCl}_{2}$ 作为催化剂还原亚砜的例子. 该体系反 应条件温和(室温下即可进行), 反应收率和化学选择性 都很出色(Eq. 8). 值得注意的是，由于二烷基亚砜的反 应性远高于二芳基亚砜，因此在催化还原二烷基亚砜时 二氯亚砜的用量要远远低于二芳基亚砜. 该方法的优势 在于其简单的操作，温和的反应条件，广泛的底物适用 性以及原料低廉的价格等.

$$
\begin{aligned}
& \mathrm{PPh}_{3}+\underset{\mathrm{R}^{-S^{\prime \prime}}}{\stackrel{\mathrm{O}}{\mathrm{R}^{\prime}}} \frac{\mathrm{SOCl}_{2}}{\mathrm{THF}, \text { r.t., } 2 \sim 4 \mathrm{~h}} \mathrm{R}^{-\mathrm{S}_{-\mathrm{R}^{\prime}}} \\
& R, R^{\prime} \text { : phenyl,benzyl,alkyl } \quad 92 \% \sim 99 \% \text { yields }
\end{aligned}
$$

2014 年, Ghorbani-Vaghei 等 $^{[20]}$ 利用 $N, N, N, N$-四溴1,3 -苯二磺酰胺 $(\mathrm{TBBDA}) / \mathrm{PPh}_{3}$ 体系在二氯甲烷中对一 系列的亚砜进行了还原，该体系的特点是反应迅速(1 $\min$ 以内), 且反应条件温和(室温下反应), 此外较高的 收率、简单的操作以及产物分离方便也是该方法的优势 所在. 作者还将 TBBDA 替换为 PBBS 对反应进行了探 究，反应并没有发生(Eq. 9).

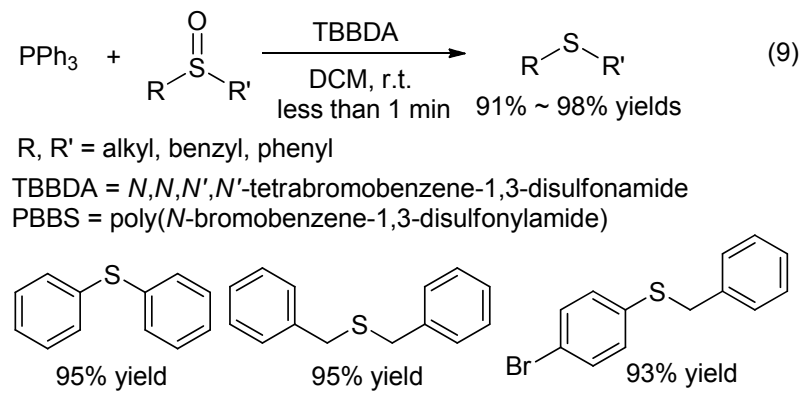

2018 年, Zhao 等 ${ }^{[21]}$ 报道了利用 $\mathrm{PCl}_{3}$ 作为还原剂对 亚砜的还原(Eq. 10). 该方法以乙腈作为溶剂, $25{ }^{\circ} \mathrm{C}$ 下 只需 $0.5 \mathrm{~h}$ 就能完成对一系列亚砜的还原. 反应条件温 和，原料便宜易得，底物适用性广。作者提出了该反应 
的机理, 并对其进行了验证: 首先, $\mathrm{PCl}_{3}$ 受到亚砜的氧 原子的攻击, 产生盐 6 , 然后盐 6 分解为硫化物 $\mathbf{5}$ 和三氯 氧磷(Scheme 4).

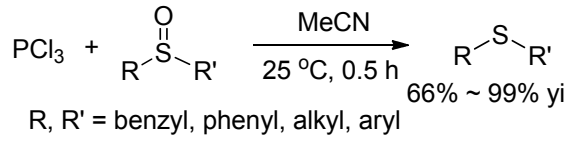

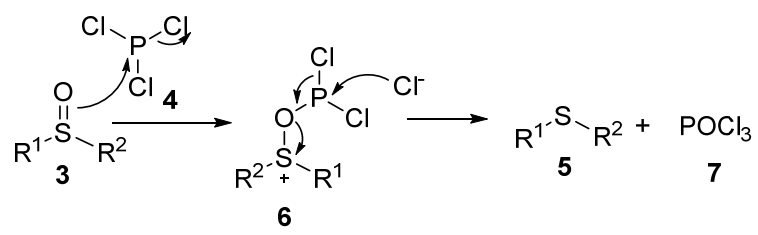

图式 $4 \mathrm{PCl}_{3}$ 还原亚砜的反应机理研究

Scheme 4 Proposed mechanism for deoxygenation of sulfoxides with $\mathrm{PCl}_{3}$

\section{2 含硼化合物还原亚砜}

含嗍试剂作为还原剂在亚砜还原领域受到很多研 究人员的青睐. 2003 年 $\mathrm{NaBH}_{4} / \mathrm{I}_{2}$ 的体系被研究人员发现 可以用来还原各种官能团, 如羧酸、羧酸酯、酰胺等. Karimi 等 ${ }^{[22]}$ 受其启发, 利用该体系对亚砜的还原进行了 尝试, 发现即使在亚砜中存在其它可还原官能团(酯、腈 和双键等)的情况下, 该体系依旧能够实现亚砜的化学 选择性脱氧还原(Eq. 11), 显示出良好的化学选择性.

$$
\begin{aligned}
& \mathrm{NaBH}_{4}+\underset{\mathrm{R}^{-\mathrm{S}_{-}}}{\stackrel{\mathrm{O}}{\mathrm{R}^{\prime}}} \stackrel{1.2 \text { or } 2 \text { equiv. } \mathrm{I}_{2}}{\text { anhyd. THF, r.t. }} \mathrm{R}^{-\mathrm{S}_{\mathrm{R}^{\prime}}} \\
& \mathrm{R}, \mathrm{R}^{\prime}=\text { phenyl, benzyl, alkyl } 18 \mathrm{~min} \quad 57 \% \sim 98 \% \text { yields }
\end{aligned}
$$

2004 年, Harrison 等 ${ }^{[23]}$ 利用 Hbcat (儿茶酚硼烷)在室 温下高效还原了一系列脂肪族亚砜和芳香族亚砜(Eq. 12), 该试剂相对于之前的含硼试剂(如 $\mathrm{BBr}_{3}$ 等)来说更 加稳定且易于处理. 不过在还原某些含有吸电子基或大 位阻基团的亚砜时, 反应速度十分缓慢, 作者通过添加 过量的儿茶酚嗍烷(或加入铑作为催化剂)很好地解决了 该问题.

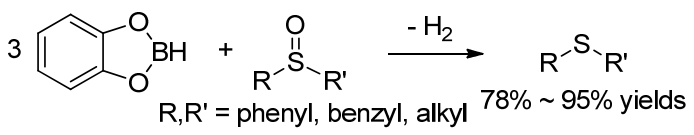

2006 年, Roy 研究小组 ${ }^{[24]}$ 发展了一种条件温和、通 用有效的还原亚砜的方法. 他们以 $\mathrm{HBBr}_{2} / \mathrm{Me}_{2} \mathrm{~S}$ 体系化 学选择性还原了各种脂肪族亚砜和芳香族亚砜(Eq. 13), 该方法的反应时间短且收率高.

$$
\begin{aligned}
& \mathrm{HBBr}_{2}+\underset{\mathrm{R}^{-} \mathrm{S}_{-\mathrm{R}^{\prime}}}{\mathrm{O}} \frac{\mathrm{Me}_{2} \mathrm{~S}}{\mathrm{DCM}, 0 \sim 25^{\circ} \mathrm{C}, 10 \sim 15 \mathrm{~min}} \mathrm{R}^{-S^{-} \mathrm{R}^{\prime}} \\
& R, R^{\prime}=\text { phenyl, benzyl, alkyl }
\end{aligned}
$$

2007 年, Fernandes 和 Romao ${ }^{[25]}$ 报道了 HBcat(儿茶 酚嗍烷 $) / \mathrm{MoO}_{2} \mathrm{Cl}_{2}\left(\mathrm{H}_{2} \mathrm{O}\right)_{2}$ 和 $\mathrm{BH}_{3}-\mathrm{THF} / \mathrm{MoO}_{2} \mathrm{Cl}_{2}$ 体系在非 极性介质中对一系列亚砜的还原(Eq. 14), 解决了之前 Harrison 等利用 HBcat 对亚砜进行还原时遇到的反应速 化剂在还原过程中是否具有积极作用, 在没有添加催化 剂的情况下于体系中加入 2 equiv. 的儿茶酚硼烷, 结果 要么硫醚产物收率非常低, 要么反应根本没有发生. 在 作者向体系中补加一定量的催化剂之后，可以观察到还 原反应的速率得到明显提升.

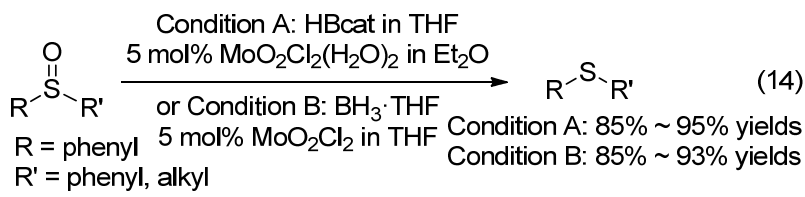

2010 年, Zhang 等 ${ }^{[26]}$ 利用 $\mathrm{HfCl}_{4} / \mathrm{KBH}_{4}$ 体系实现了对 各类二烷基、二芳基和芳基烷基亚砜的还原. 在此前工 作中，他们还利用该体系顺利还原了各类羧酸及其衍生 物. 该体系以四氢呋喃为溶剂, 回流温度下于氮气的环 境中进行反应. 当亚砜、 $\mathrm{HfCl}_{4}$ 和 $\mathrm{KBH}_{4}$ 的比例为 0.8 : $1.0: 4.0$ 时, 反应达到最佳条件(Eq. 15). 并且带有吸电 子基团的亚砜比带有给电子基团的亚砜反应速度更快. 此外作者还利用该体系对二硫化二苯并噻唑、对甲苯磺 酸、环丁砜和二苯砜等物质进行了还原, 不过遗憾的是, 只有二硫化二苯并噻唑能够发生反应.

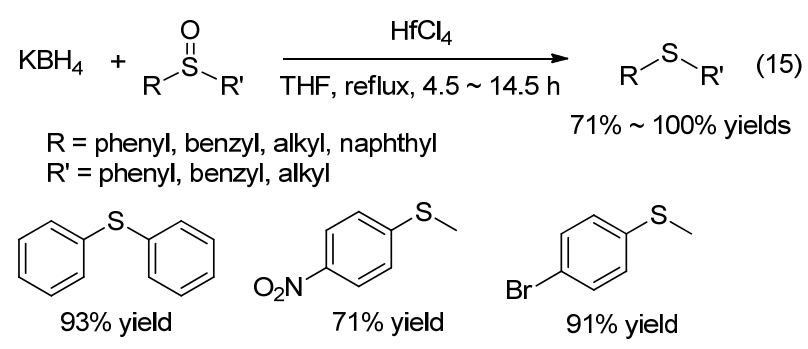

同年, Fernandes 等 ${ }^{[27]}$ 继续使用 HBcat 作为还原剂, 并在 $\mathrm{HBcat} / \mathrm{ReIO}_{2}\left(\mathrm{PPh}_{3}\right)_{2} 、 \mathrm{HBcat} / \mathrm{Re}_{2} \mathrm{O}_{7}$ 和 $\mathrm{HBcat} / \mathrm{MTO}$ 三个体系中成功实现了对亚砜的还原. 这些体系共同的 优点有, 催化剂用量低(1 mol\%), 反应速率快(30 min 以 内)以及催化剂在空气和水中的稳定性等(Eq. 16). 不过 还原剂的过度使用 (2 equiv. 的硼烷作为还原剂) 是该反 应的不足.

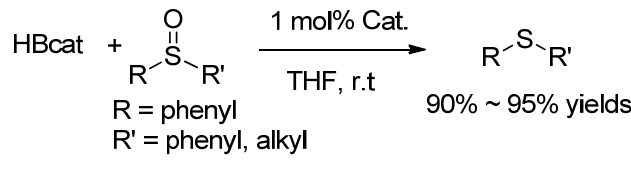

$$
\begin{aligned}
& \text { Cat.: } \mathrm{ReIO}_{2}\left(\mathrm{PPh}_{3}\right)_{2}, \mathrm{Re}_{2} \mathrm{O}_{7}, \mathrm{MTO} \cdot \mathrm{DFT}
\end{aligned}
$$
率缓慢的问题. 随后作者为了验证 $\mathrm{MoO}_{2} \mathrm{Cl}_{2}\left(\mathrm{H}_{2} \mathrm{O}\right)_{2}$ 等催 
2011 年, Yakabe 等 ${ }^{[28]}$ 在研究了硼氢化钠/氧化铝体 系对一系列醛和酮的还原反应之后, 发现所得还原产物 醇的反应收率十分优异, 并且回收的氧化铝可以重复使 用几次而反应活性不会损失. 因此作者继续利用该体系 对亚砜的还原反应进行尝试, 经过研究发现该体系在六 水合二氯化钴催化的情况下, 可以成功地对一系列亚砜 进行还原, 并且操作简单, 安全性好, 反应收率中等到 优秀(Eq. 17).

$$
\begin{aligned}
& \mathrm{NaBH}_{4}+\underset{\mathrm{R}^{-}}{\stackrel{\mathrm{O}}{\text { II }}-\mathrm{R}^{\prime}} \frac{\mathrm{CoCl}_{2} \cdot 6 \mathrm{H}_{2} \mathrm{O}}{\text { moist alumina }} \underset{\text { hexane, } 30 \sim 60^{\circ} \mathrm{C}, 3 \mathrm{~h}}{R^{-}} \mathrm{S}_{\mathrm{R}^{\prime}} \\
& \mathrm{R}, \mathrm{R}^{\prime}=\text { benzyl, alkyl, phenyl }
\end{aligned}
$$

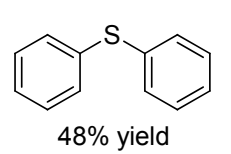

2012 年, Enthaler 等 ${ }^{[29]}$ 选用三氟甲烷磺酸锌 $\left[\mathrm{Zn}(\mathrm{OTf})_{2}\right]$ 作为催化剂, 嗍烷作为还原剂对亚砜的还原 反应进行了研究(Eq. 18). 通过条件篮选, 他们发现利用 儿茶酚硼烷和频哪醇硼烷作为还原剂时产物收率优秀, 而利用 $\mathrm{BH}_{3}$ 作为还原剂时产物只能获得中等收率. 此 外, 芳基亚砜的产率在某种程度低于烷基亚砜.

$$
\text { borane + }
$$
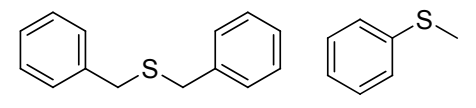

同年, Enthaler ${ }^{[30]}$ 又报道了 $\mathrm{Zn}(\mathrm{OTf})_{2} / \mathrm{B}(\mathrm{Pin})_{2}$ 对各类 脂肪族亚砜和芳香族亚砜的还原, $\mathrm{Zn}(\mathrm{OTf})_{2} / \mathrm{B}(\mathrm{Pin})_{2}$ 催化 还原体系具有较高的官能团容忍性以及较好的化学选 择性. 为了阐明锌在该反应当中的重要作用, 作者将 $\mathrm{Zn}(\mathrm{OTf})_{2}$ 替换为催化量的三氟甲磺酸 (其可能通过 $\mathrm{Zn}(\mathrm{OTf})_{2}$ 的分解形成), 没有观察到硫醚产物的生成(Eq. 19).

\section{3 卤化物还原亚砜}

2000 年, Shimizu 等 ${ }^{[31]}$ 发现四碘化钛可以使频哪醇 发生偶联反应, 以四碘化钛作为还原剂对一系列亚砜

$$
[\mathrm{B}(\mathrm{Pin})]_{2}+\underset{\mathrm{R}^{-}}{\stackrel{\mathrm{O}}{\mathrm{S}}{ }_{\mathrm{R}^{\prime}}} \frac{5 \mathrm{~mol} \% \mathrm{Zn}(\mathrm{OTf})_{2}}{\text { mesitylene, } 160^{\circ} \mathrm{C}, 48 \mathrm{~h}} \mathrm{R}^{-\mathrm{S}_{\backslash} \mathrm{R}^{\prime}}
$$

$\mathrm{R}, \mathrm{R}^{\prime}$ = alkyl, benzyl, phenyl<smiles>c1ccc(Sc2ccccc2)cc1</smiles>

$83 \%$ yield<smiles>Clc1ccc(Sc2ccc(Cl)cc2)cc1</smiles>

$98 \%$ yield $83 \% \sim 98 \%$ yields<smiles>CSc1ccccc1</smiles>

$95 \%$ yield

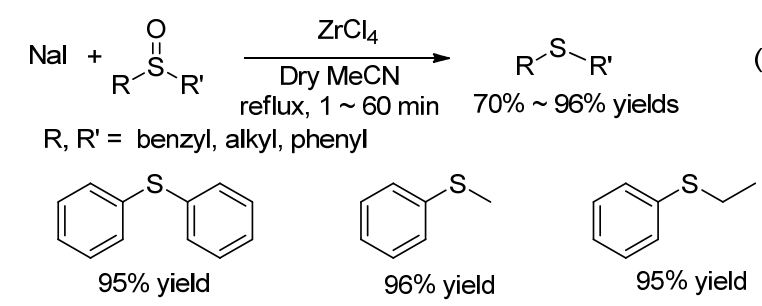

进行考察，实现了对各类芳基烷基亚砜的还原，该反应 具有良好的化学选择性，反应条件十分温和，且反应时 间短(Eq. 20).

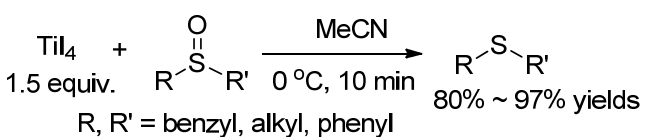<smiles>c1ccc(Sc2ccccc2)cc1</smiles>

$93 \%$ yield<smiles>OCCSc1ccccc1</smiles>

$87 \%$ yield<smiles>C#CCSc1ccccc1</smiles>

92\% yield
2005 年, Firouzabadi 等 ${ }^{[32]}$ 利用 $\mathrm{ZrCl}_{4} / \mathrm{NaI}$ 和 $\mathrm{ZrOCl}_{2}$ • $8 \mathrm{H}_{2} \mathrm{O} / \mathrm{NaI}$ 两种体系对亚砜进行了化学选择性的还原. 锆(IV)化合物作为潜在的绿色催化剂具有毒性较低、易 于处理、高活性等性质, 因此作者选用了 $\mathrm{ZrCl}_{4}$ 和 $\mathrm{ZrOCl}_{2} \bullet 8 \mathrm{H}_{2} \mathrm{O}$ 两种安全可商购的锆化合物作为该反应的 催化剂. 在无水乙腈中回流反应体系, 以中等到优秀的 收率还原了一系列芳香族和脂肪族亚砜, 且 $\mathrm{ZrCl}_{4} / \mathrm{NaI}$ 体系的反应速率要快于 $\mathrm{ZrOCl}_{2} \bullet 8 \mathrm{H}_{2} \mathrm{O} / \mathrm{NaI}$ 体系(Eq. 21).

2008 年, Ternois 等 ${ }^{[33]}$ 利用 $\mathrm{KI} / \mathrm{AcCl}$ 体系, 在酸性介 质中化学选择性地还原了几类含有其它可还原官能团 的亚砜(Eq. 22), 反应速率很快(30 $\mathrm{min}$ 以内)且收率优 秀.

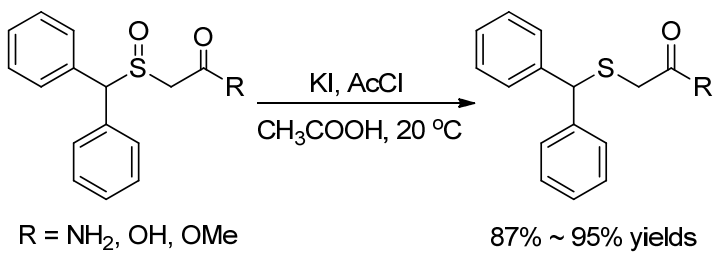

同年 Bahrami 等 ${ }^{[34]}$ 报道了 $\mathrm{Tf}_{2} \mathrm{O}$ (三氟甲磺酸酐)和 $\mathrm{KI}$ 组成的体系对一系列脂肪族和芳香族亚砜的还原 (Eq. 23), 该体系反应条件温和且收率优秀, 反应速率较 快 (6 min 以内). 在酯、醛、酸等可还原官能团的存在下 亚砜依然能够选择性脱氧还原成硫醚, 体现出很好的官 能团容忍性.

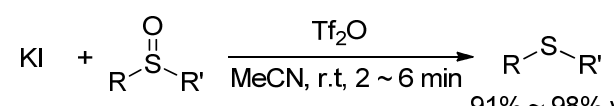

$$
\begin{aligned}
& \mathrm{R}=\text { phenyl, alkyl, allyl } \\
& \mathrm{R}^{\prime}=\text { phenyl, benzyl, alkyl, allyl }
\end{aligned}
$$


该反应的机理在 Scheme 5 中给出. 首先, 三氟甲磺 酸酐与亚砜的氧原子进行配位使得碘负离子的进攻成 为可能, 所产生的碘化物随后又被另一个碘负离子进 攻, 得到脱氧的硫化物.

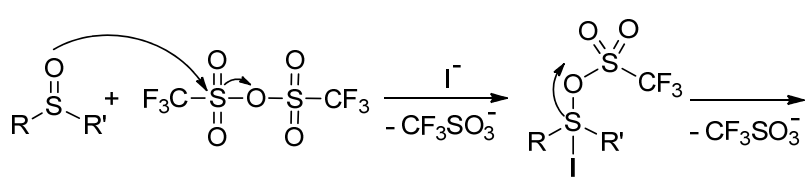

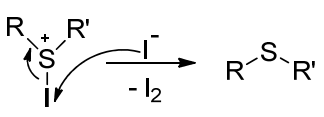

图式 $5 \mathrm{KI} / \mathrm{Tf}_{2} \mathrm{O}$ 还原亚砜的反应机理研究

Scheme 5 Deoxygenation of sulfoxides using the $\mathrm{KI} / \mathrm{Tf}_{2} \mathrm{O}$ system

2010 年 Bahrami 等 ${ }^{[35]}$ 发现在 $1,3,5$-三唑-2,4,6-三磷 酸-2,2,4,4,6,6-四氯化钾(TPAC)/KI 体系下, 亚砜能够迅 速脱氧还原成相应的硫醚(Eq. 24), 反应时间很短, 产物 硫醚收率优秀(能达到 $98 \%$ 以上分离收率). 此外该反应 不需要溶剂就能发生, 减少了对有机溶剂的使用以及其 他废物的形成, 绿色和环保是该体系一个很显著的优 点.

$$
\begin{aligned}
& \mathrm{KI}+\underset{\mathrm{R}^{-}}{\stackrel{\mathrm{II}}{\mathrm{S}}{ }_{\mathrm{R}^{\prime}}} \stackrel{\text { TPAC }}{\stackrel{\text { solvent-free, } 1 \sim 5 \mathrm{~min}}{\longrightarrow}} \mathrm{R}^{-\mathrm{S}_{-\mathrm{R}^{\prime}}} \\
& \mathrm{R}=\text { phenyl, alkyl, benzyl, naphthyl } \\
& \mathrm{R}^{\prime}=\text { phenyl, alkyl, benzyl }
\end{aligned}
$$

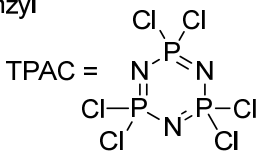

2011 年, Bahrami 等 ${ }^{[36]}$ 采用 $\mathrm{H}_{2} \mathrm{O}_{2} / \mathrm{TCT}$ (三聚氯氰)体 系以出色的收率将一系列硫醚化合物氧化成了对应的 砜类化合物, 作者为了证明 $\mathrm{TCT}$ (三聚氯氰)的高效性与 适用性, 又利用 $\mathrm{KI} / \mathrm{TCT}$ 体系对亚砜进行了还原反应的 研究, 并最终以温和的条件(乙腈中室温下反应), 在较 短的反应时间内(小于 $15 \mathrm{~min}$ )还原了一系列烷基和芳基 亚砜. 该方法操作简单, 具有很好的官能团容忍性, 产 物的分离也十分容易(Eq. 25).

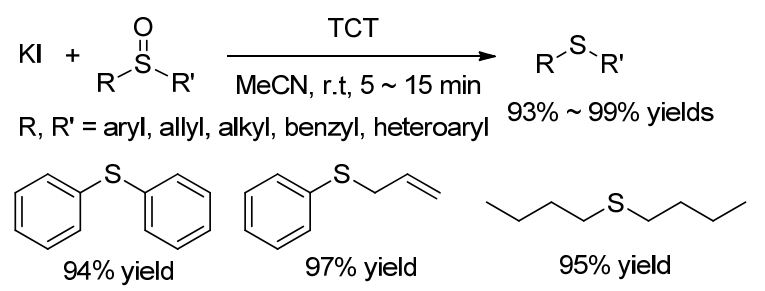

2013 年, Zarei 等 ${ }^{[37]}$ 利用碘化钠/硅胶负载硫酸体系 在聚乙二醇(PEG-200)中以出色的收率实现了对各类二 烷基、二芳基、芳基烷基和烯丙基亚砜的还原(Eq. 26).
反应对环境比较友好且后处理相对容易，因此该反应体 系可以适用于大规模反应. 此外，就反应时间、产率和 温度而言，该体系比其他基于 $\mathrm{Na}(\mathrm{K}) \mathrm{I} /$ 酸的还原体系 $\left(\mathrm{K}(\mathrm{Na}) \mathrm{I} / \mathrm{TsOH}, \mathrm{KI} / \mathrm{HClO}_{4}, \mathrm{KI} / \mathrm{HCl}\right.$ 等)更为优秀.

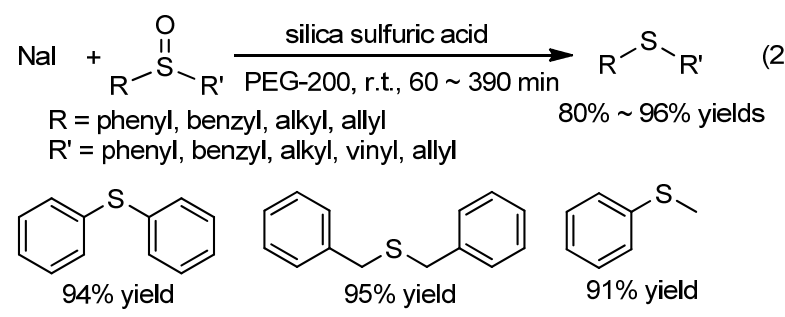

2014 年, Atabaki 等 ${ }^{[38]}$ 发现碳质固体酸(CSA)在碘化 钠作为还原剂的条件下, 以良好到优秀的收率催化了一 系列亚砜的还原(Scheme 6). 该体系的优势在于其温和 的反应条件、低价环保的原料以及简单的后处理工序. 此外, 在双氧水作为氧化剂的情况下碳质固体酸(CSA) 还能催化硫醚氧化为亚砜.

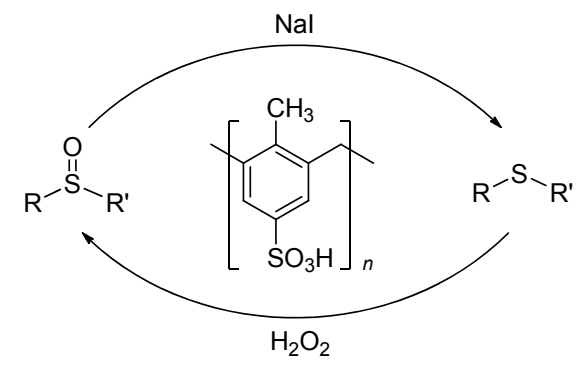

图式 6 利用碳质固体酸和碘化钠还原亚砜

Scheme 6 Deoxygenation of sulfoxides with CSA and NaI

同年, Mohanazadeh 等 ${ }^{[39]}$ 利用硅溴化合物(SB)在室 温下于二氯甲烷中还原了一系列脂肪族和芳香族亚砜, 该方法优势在于其优异的产率、多样化的条件、简单的 操作以及易于分离的产物(Eq. 27).

2017 年, $\mathrm{TaCl}_{5}$ 强烈的亲氧特性以及较低的毒性吸 引了 Yoo 小组的研究人员 ${ }^{[40]}$ 选用其作为研究亚砜还原 反应的催化剂(Eq. 28), 并在 $\mathrm{NaI}$ 作为反应的还原剂的条 件下，以较高的收率在室温下还原了一系列亚砜.

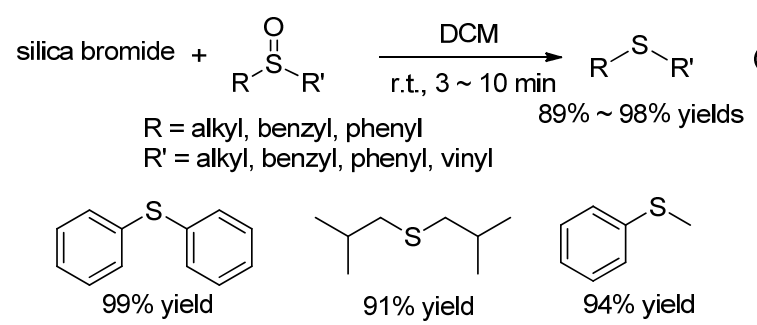

$$
\begin{aligned}
& \mathrm{Nal}+\underset{R^{-}}{\stackrel{\mathrm{II}}{\mathrm{S}}{ }_{\mathrm{R}^{\prime}}} \stackrel{\mathrm{TaCl}_{5}}{\mathrm{MeCN} \text {, r.t., 3 } \sim 5 \mathrm{~min}} \mathrm{R}^{-\mathrm{S}_{-}} \mathrm{R}^{\prime} \\
& \mathrm{R}, \mathrm{R}^{\prime}=\text { alkyl, aryl } \quad 89 \% \sim 96 \% \text { yields }
\end{aligned}
$$


该反应的时间较短( $3 \sim 5 \mathrm{~min})$, 对硝基、醛基、甲氧 基、卤素等官能团具有较好的容忍性. 该还原过程涉及 的反应机理如 Scheme 7 所示. 首先, $\mathrm{TaCl}_{5}$ 与亚砜的氧原 子进行配位, 使得碘负离子的进攻成为可能, 所产生的 碘化物随后又被另外的碘负离子进攻得到脱氧的硫化 物与单质 $\mathrm{I}_{2}$.

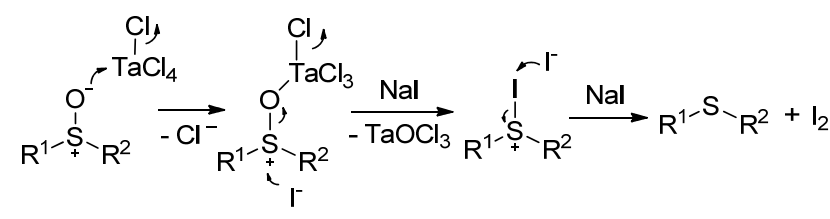

图式 $7 \mathrm{TaCl}_{5} / \mathrm{NaI}$ 体系还原亚砜的反应机理研究 Scheme 7 Proposed mechanism for the deoxygenation of sulfoxide using $\mathrm{TaCl}_{5} / \mathrm{NaI}$

\section{4 含硫化合物还原亚砜}

2003 年, Karimi 等 ${ }^{[41]}$ 在探索中性或近中性催化剂的 潜在用途过程中发现, $N$-溴代丁二酰亚胺(NBS)或 $\mathrm{I}_{2}$ 在 硫醇作为还原剂的情况下, 能够很好地催化亚砜还原反 应的发生. 作者对还原剂硫醇进行了篮选, 最终选择以 3-颈基丙酸(该分子中存在的 $\mathrm{CO}_{2} \mathrm{H}$ 基团可以在后处理 阶段通过简单的 $\mathrm{NaOH}$ 水溶液洗涤, 方便地分离出副产 物)作为该体系的还原剂. 该方法反应条件温和, 反应 时间短，具有良好的化学选择性(Eq. 29).

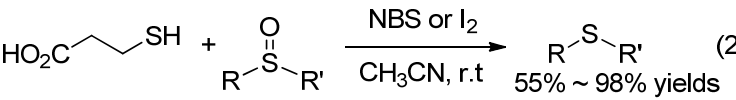

$$
\begin{aligned}
& \mathrm{R}, \mathrm{R}^{\prime}=\text { phenyl, benzyl, aryl, alkyl, allyl }
\end{aligned}
$$

同年, Karimi 等 ${ }^{[42]}$ 利用 3-颈基丙酸在 10 $20 \mathrm{~mol} \%$ 三甲基氯硅烷的催化下成功还原了各类烷基和芳基亚 砜，该反应条件温和且后处理简单(Eq. 30).

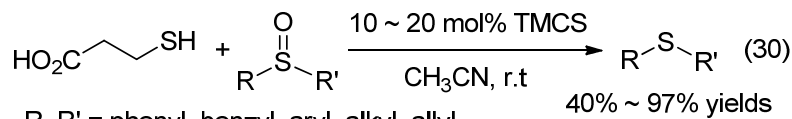

$\mathrm{R}, \mathrm{R}^{\prime}=$ phenyl, benzyl, aryl, alkyl, allyl

2012 年, Jabbari 等 ${ }^{[43]}$ 报道了利用硫代乙酸作为还原 剂, 碘作为催化剂对亚砜的还原. 在此之前已经有过关 于硫代酸还原亚砜相关的报道，不过其反应时间长，产 率低等缺点导致该方法没有得到广泛的应用. 然而, 考 虑到硫代酸是一种廉价且常用的实验室试剂, 作者希望 能够在 $\mathrm{I}_{2}$ 催化下利用硫代乙酸实现亚砜的高效脱氧还 原. 最终在室温下以优秀的收率还原了苄基、烯丙基、 烷基和芳基亚砜等一系列亚砜(Eq. 31). 与其他使用硫

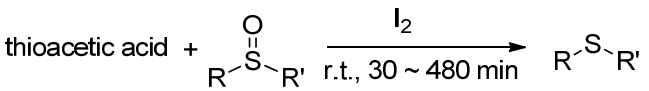

$$
\begin{aligned}
& R=\text { phenyl, benzyl, alkyl } \quad 75 \% \sim 98 \% \text { yields } \\
& \mathrm{R}^{\prime} \text { = phenyl, benzyl, alkyl, vinyl }
\end{aligned}
$$

代酸试剂的还原方法相比，该方法反应速率更快且产率 更高, 反应机理如 Scheme 8 所示.

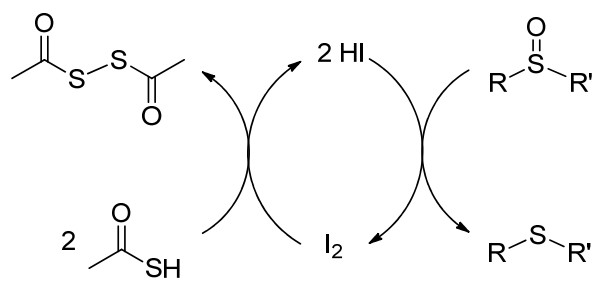

图式 8 硫代乙酸还原亚砜的反应机理研究

Scheme 8 Proposed mechanism for deoxygenation of sulfoxides with thioacetic acid

2015 年, Abbasi 等 ${ }^{[44]}$ 报道了 $\mathrm{NaHSO}_{3} / \mathrm{I}_{2}$ 体系对亚砜 的还原, 其中 $\mathrm{I}_{2}$ 作为催化剂, $\mathrm{NaHSO}_{3}$ 作为还原剂, 反应 在室温或 $50{ }^{\circ} \mathrm{C}$ 下于氯仿中以优秀的收率还原了一系列 亚砜(Eq. 32). 该反应的官能团容忍性良好，在可被还原 官能团(包括醛基、硝基等)的存在下，亚砜依旧成功还 原而没有产率损失. 较高的产率、温和的反应条件、简 单的实验操作以及廉价的反应试剂等都提高了该方法 的实用性, 反应机理如 Scheme 9 所示.

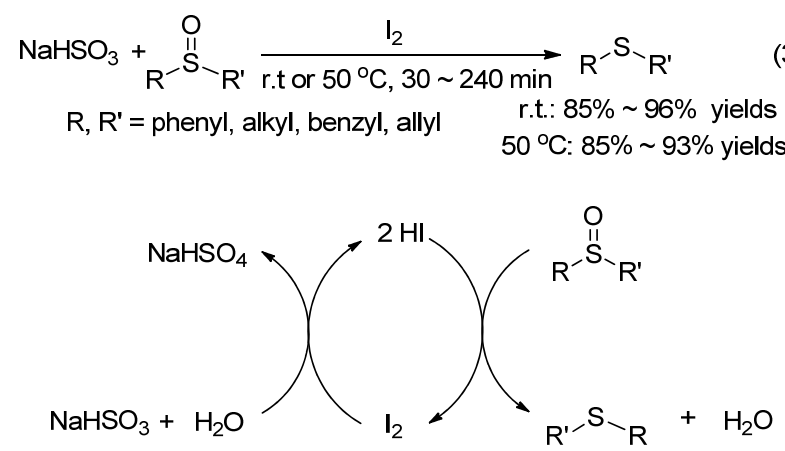

图式 $9 \mathrm{NaHSO}_{3} / \mathrm{I}_{2}$ 体系还原亚砜反应机理研究

Scheme 9 Proposed mechanism for reduction of sulfoxides with the $\mathrm{NaHSO}_{3} / \mathrm{I}_{2}$ system

2016 年, $\mathrm{Sanz}$ 等 ${ }^{[45]}$ 希望在之前利用 $\mathrm{MoO}_{2} \mathrm{Cl}_{2}(\mathrm{dmf})_{2}$ 作为催化剂, 频哪醇和甘油等作为还原剂还原亚砜的反 应基础上，继续寻找一些对环境友好且可持续使用的还 原剂. 他们在探究过程中发现 3-颈丙基硅胶(MPS)作为 一种固体负载的还原剂, 可以在较短的时间内以较高的 收率对一系列亚砜进行还原(Eq. 33), 并且反应的纯化 过程仅需要进行过滤, 而不需要用到萃取或者层析过柱 等方法.

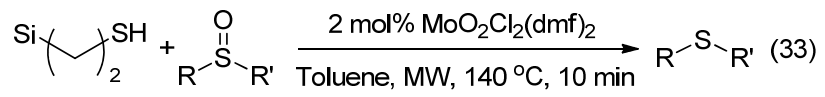

$\mathrm{R}, \mathrm{R}^{\prime}=$ alkyl, phenyl, aryl

$67 \% \sim 97 \%$ yields

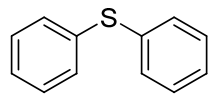

$94 \%$ yield
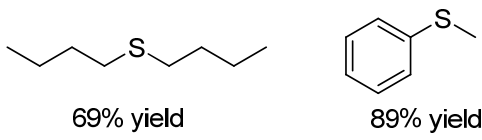

$89 \%$ yield 


\section{5 含硅化合物还原亚砜}

2006 年, Fernandes 和 Romao 等 ${ }^{[46]}$ 利用 $\mathrm{MoO}_{2} \mathrm{Cl}_{2}$ 作 为催化剂, $\mathrm{PhSiH}_{3}$ 作为还原剂对一系列亚砜进行了还原, 反应收率高并且官能团容忍性好(Eq. 34). 随后作者继 续发展了 PMHS(聚甲基聚硅氧烷) $/ \mathrm{MoO}_{2} \mathrm{Cl}_{2}\left(\mathrm{H}_{2} \mathrm{O}_{2}\right)$ 的绿 色还原体系, 该催化体系可以在甲醇或水中进行反应, 最终以中等到优秀的收率得到相应的硫醚. 该反应体系 环境友好性，适合用来做大规模的还原反应.

$$
\begin{aligned}
& \mathrm{PhSiH}_{3}+\underset{\mathrm{R}^{-}}{\stackrel{\mathrm{II}}{\mathrm{O}}{ }_{-\mathrm{R}^{\prime}}} \frac{5 \mathrm{~mol} \% \mathrm{MoO}_{2} \mathrm{Cl}_{2}}{\text { toluene, reflux, } 2 \sim 20 \mathrm{~h}} \mathrm{R}^{-\mathrm{S}_{{ }^{\prime}} \mathrm{R}^{\prime}} \\
& \mathrm{R}=\text { butyl, phenyl } \\
& \mathrm{R}^{\prime}=\text { alkyl, alkyl acetate, phenyl }
\end{aligned}
$$

2009 年, Fernandes 和 Sousa 等 ${ }^{[47]}$ 发现 $\mathrm{PhSiH}_{3} /$ $\mathrm{ReIO}_{2}\left(\mathrm{PPh}_{3}\right)_{2}$ 体系可以在适当的条件下, 以高收率还原 一系列脂肪族和芳香族亚砜(Eq. 35), 并对很多官能团 如酯基、硝基、醛基的容忍性都很好. 该体系优点在于 其催化剂稳定性好, 用量低 ( $1 \mathrm{~mol} \%$ ), 反应迅速, 操作 简单. Scheme 10 给出了该反应的机理.

$$
\begin{aligned}
& \mathrm{PhSiH}_{3}+\underset{\mathrm{R}^{-}}{\stackrel{\mathrm{O}}{\|}{ }_{\mathrm{R}^{\prime}}} \frac{\mathrm{RelO}_{2}\left(\mathrm{PPh}_{3}\right)_{2}}{\text { THF, r.t., } 15 \sim 135 \mathrm{~min}} \mathrm{R}^{-\mathrm{S}_{-\mathrm{R}^{\prime}}} \\
& R=\text { phenyl, butyl } \\
& \mathrm{R}^{\prime}=\text { phenyl, alkyl, benzyl, alkyl acetate }
\end{aligned}
$$

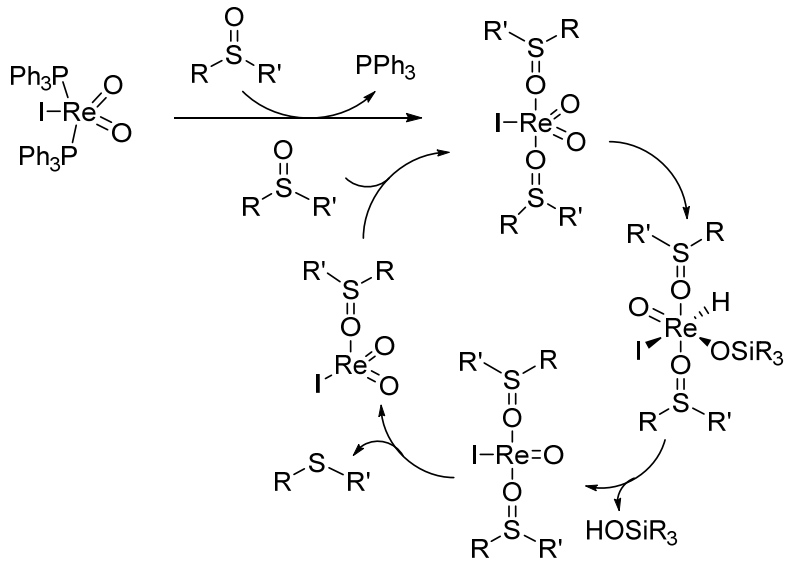

图式 $10 \mathrm{PhSiH}_{3} / \mathrm{ReIO}_{2}\left(\mathrm{PPh}_{3}\right)_{2}$ 体系还原亚砜机理研究 Scheme 10 Proposed mechanism for sulfoxide deoxygenation with the $\mathrm{PhSiH}_{3} / \mathrm{ReIO}_{2}\left(\mathrm{PPh}_{3}\right)_{2}$ system

2010 年, Fernandes ${ }^{[48]}$ 继续报道了 $\mathrm{PhSiH}_{3} / \mathrm{HReO}_{4}$ 体 系与 $\mathrm{HBcat} / \mathrm{HReO}_{4}$ 体系对亚砜的还原, 这两种体系都可 以在非惰性的条件下进行反应, 并且都具有很好的化学 选择性以及官能团容忍性. 在对比两个体系的过程中, 可以发现 $\mathrm{HBcat} / \mathrm{HReO}_{4}$ 体系的反应速率要快于 $\mathrm{PhSiH}_{3} /$ $\mathrm{HReO}_{4}$ 体系. 此外催化剂 $\mathrm{HReO}_{4}$ 的空气稳定性以及较为 低廉的价格是该方法的优势之一(Eq. 36).

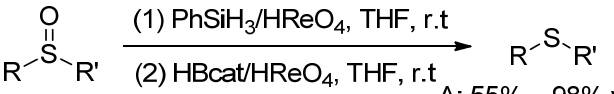

$$
\begin{aligned}
& \begin{array}{ll}
R, R^{\prime}=\text { phenyl, alkyl, benzyl } & \text { B: } 55 \% \sim 98 \% \text { yields } \\
& \text { B: } 66 \% \sim 98 \% \text { yields }
\end{array}
\end{aligned}
$$

2011 年, Enthaler ${ }^{[49]}$ 以对甲苯基亚砜作为模板底物, $\mathrm{PhSiH}_{3}$ 作为还原剂直接对其进行还原时几乎没有硫醚 产物的生成. 于是作者又加入了 $5 \mathrm{~mol} \%$ 的 $\mathrm{Zn}(\mathrm{OTf})_{2}$ 对 反应进行催化，结果在非惰性条件下以 $99 \%$ 以上的收率 得到了相应的还原产物，随后作者利用该体系对底物进 行了拓展, 并以优秀的收率还原了一系列的脂肪族和芳 香族亚砜(Eq. 37).

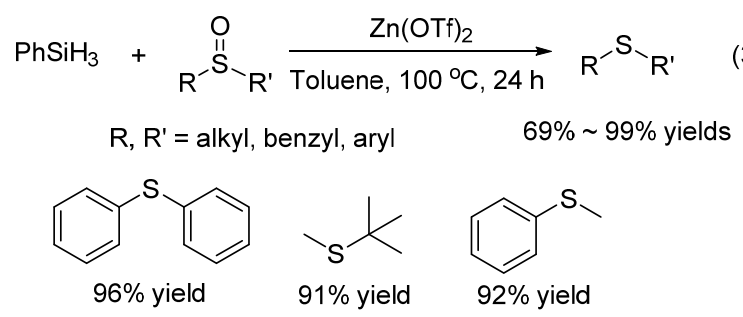

同年 Enthaler 和 Weiduer ${ }^{[50]}$ 报道了以乙酰丙酮铜(II) 作为催化剂, 二苯基硅烷作为还原剂对亚砜的还原(Eq. 38). 作者详细研究了不同反应参数对反应催化活性的 影响，在各种亚砜的还原中得出了该催化体系的一些应 用范围和限制. 值得注意的是，在含有酯、酰胺、砜和 含氧官能团的添加剂存在下，亚砜可以被选择性地还原 为硫化物, 而添加剂的官能团不受影响. 但在某些情况 下，与没有添加剂的反应相比，硫化物的产率有一定程 度降低, 不过大多数情况下该反应都能获得高转化率和 优异的化学选择性.

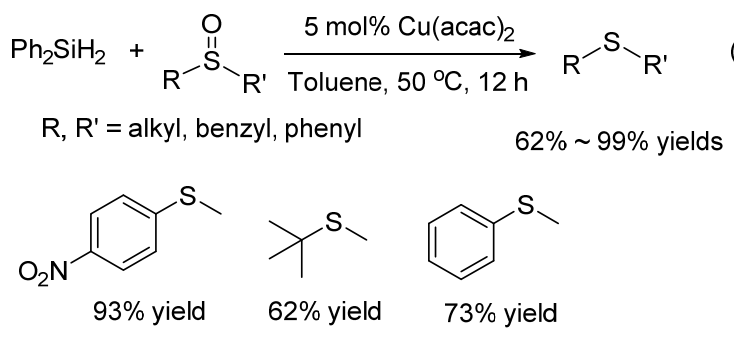

随后 Enthaler ${ }^{[51]}$ 又报道了利用 $\left[\mathrm{Fe}_{2}(\mathrm{Co})_{9}\right]$ 作为催化剂 对亚砜的还原. 相比于之前利用铁络合物对 $\mathrm{C}=\mathrm{O} 、 \mathrm{C}=$ $\mathrm{C} 、 \mathrm{C}=\mathrm{N}$ 和 $\mathrm{NO}_{2}$ 的还原，几乎没有人研究过铁络合物催 化亚砜的还原. 作者首次利用 $\left[\mathrm{Fe}_{2}(\mathrm{Co})_{9}\right]$ 作为催化剂, $\mathrm{PhSiH}_{3}$ 或聚甲基聚硅氧烷(PMHS)作为还原剂于非惰性 的条件下在甲苯中还原了一系列的芳香族和脂肪族亚 砜，该方法的优点在于各种底物还原过程中的优异产率 和良好的官能团容忍性(Eq. 39). 


$$
\begin{aligned}
& \underset{\text { or PMHS }}{\mathrm{PhSiH}_{3}}+\underset{\mathrm{R}^{-S_{-}}}{\stackrel{\mathrm{II}}{\mathrm{R}^{\prime}}} \frac{\left[\mathrm{Fe}_{2}(\mathrm{Co})_{9}\right]}{\text { toluene, } 100^{\circ} \mathrm{C}, 24 \mathrm{~h}} \mathrm{R}^{-} \mathrm{S}_{\mathrm{R}^{\prime}} \\
& R, R^{\prime}=\text { benzyl, alkyl, phenyl }
\end{aligned}
$$

2014 年, Sousa 等 ${ }^{[52]}$ 报道了以含有杂环配体的氧代铼配合物 $\left[\operatorname{ReOBr}_{2}(\mathrm{hmpbta})\left(\mathrm{PPh}_{3}\right)\right]$ 作为催化剂还原亚砜 的方法, 该方法采用硅烷或硼烷作为还原剂, 其中频哪 醇嗍烷和 $\mathrm{PhSiH}_{3}$ 是该方法最高效的两类还原剂(Eq. 40), 这两类体系的优点在于低催化剂用量(1 mol \%), 较短的 反应时间以及催化剂的稳定性等. 该反应的机理见 Scheme 11: 首先一分子亚砜取代 $\left[\operatorname{ReOBr}_{2}\right.$ (hmpbta)$\left.\left(\mathrm{PPh}_{3}\right)\right]$ 中的 $\mathrm{PPh}_{3}$ 生成 $\left[\mathrm{ReOBr}_{2}(\mathrm{hmpbta})\left(\mathrm{R}_{2} \mathrm{SO}\right)\right]$, 接下来 还原剂(硅烷或嗍烷)与之反应生成 $\mathrm{ReH}(\mathrm{OR}) \mathrm{Br}_{2}-$ $($ hmpbta $)\left(\mathrm{R}_{2} \mathrm{SO}\right)$, 随着硫化物和一分子 $\mathrm{HOR}(\mathrm{R}=\mathrm{Bpin}$ 或 $\mathrm{SiPhH}_{2}$ ) 释放得到 $\mathrm{ReOBr}_{2}(\mathrm{hmpbta})$, 亚砜的还原也随 之完成. 最后, 新的亚砜分子与金属中心配位, 再生成 氧一锞络合物 $\left[\mathrm{ReOBr}_{2}(\mathrm{hmpbta})\left(\mathrm{R}_{2} \mathrm{SO}\right)\right]$.

$$
\begin{aligned}
& \text { Condition A: } \mathrm{PhSiH}_{3} / 1 \mathrm{~mol} \%\left[\mathrm{ReOBr}_{2}(\mathrm{hmpbta})\left(\mathrm{PPh}_{3}\right)\right] \\
& \mathrm{R}^{-\mathrm{II}_{-}} \mathrm{R}^{\prime} \frac{\mathrm{THF} \text {, reflux, } 10 \sim 120 \mathrm{~min}}{\stackrel{\text { Condition B: HBPin/1 mol\% }\left[\mathrm{ReOBr}_{2}(\mathrm{hmpbta})\left(\mathrm{PPh}_{3}\right)\right]}{\longrightarrow}} \\
& \mathrm{R}, \mathrm{R}^{\prime} \text { = benzyl, alkyl, aryl } \\
& \text { THF, reflux, } 15 \sim 60 \mathrm{~min} \\
& \mathrm{R}^{-\mathrm{S}}-\mathrm{R}^{\prime} \\
& \text { Condition A: } 76 \% \sim 95 \% \text { yields } \\
& \text { Condition B: } 70 \% \sim 98 \% \text { yields }
\end{aligned}
$$

2017 年, 硼路易斯酸作为活化氢硅烷和相关转化 的有力试剂引起了研究人员的广泛兴趣. 同时, 低毒、 高活性和易处理的氢硅烷也受到了相当多的关注, 于是 Ding 等 ${ }^{[53]}$ 在 $5 \mathrm{~mol} \%$ 的 $\mathrm{B}\left(\mathrm{C}_{6} \mathrm{~F}_{5}\right)_{3}$ 的催化下, 利用苯基硅 烷对一系列亚砜进行还原(Eq. 41). 该方法在构建药物 中间体和天然产物时显示出高化学选择性和良好的官 能团容忍性, 在其他可还原官能团存在下依然能够选择 性地还原亚砜, 并且反应条件温和, 收率较高. 此外该 方法对于一些胺的 $N$-氧化物也能够进行还原.

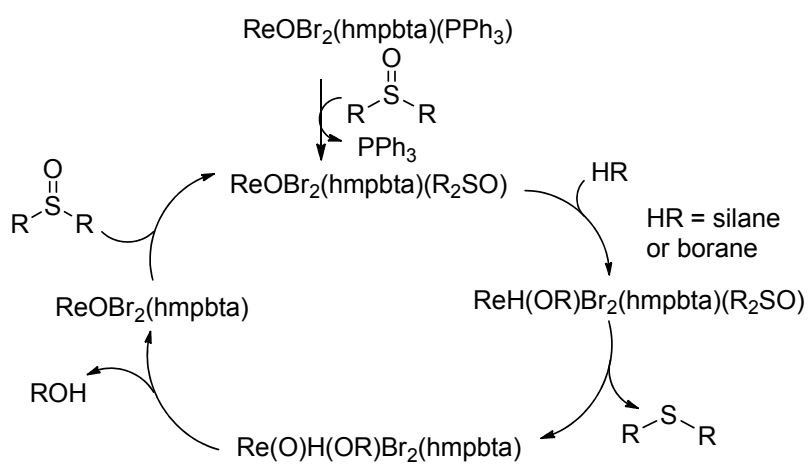

图式 $11 \mathrm{PhSiH}_{3}$ 和 $\mathrm{HBpin}$ 还原亚砜反应机理研究

Scheme 11 Proposed mechanism for the deoxygenation of sulfoxides with $\mathrm{PhSiH}_{3}$ or HBpin as the reducing agent

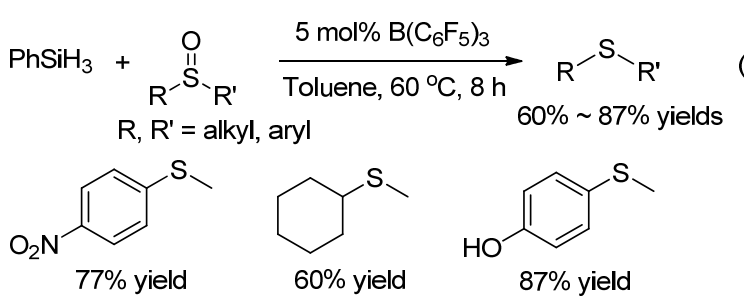

2017 年, Oestreich 等 ${ }^{[54]}$ 在利用三乙基硅氢和 $\mathrm{B}\left(\mathrm{C}_{6} \mathrm{~F}_{5}\right)_{3}(10 \mathrm{~mol} \%)$ 还原各类硝基化合物的还原体系基 础上，继续对该反应体系进行探究，发现各类烷基芳 基、二烷基以及二芳基亚砜和少量砜类化合物也能在该 体系下得到还原. 该方法反应条件温和，但三乙基硅氢 的过量使用以及较差的官能团容忍性导致该方法的应 用受到限制(Eq. 42).

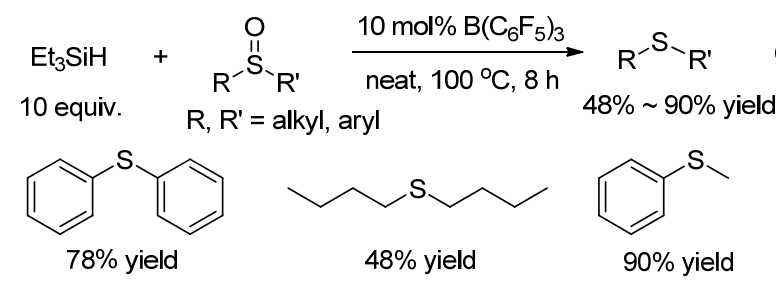

\section{6 金属及其化合物还原亚砜}

有机金属化学为有机合成开辟了一个新的篇章, 很 多金属及其化合物所参与的亚砜还原方法已经接连被 各个研究小组所报道. 2001 年 Firouzabadi 等 ${ }^{[55]}$ 以五氯化 钼作为催化剂, 锌粉作为还原剂在无水四氢呋喃(THF) 中(或者以 $\mathrm{NaI}$ 为还原剂在干燥的乙腈中)以高产率将亚 砜转化为相应的硫醚, 该方法后处理简单并且反应收率 较高(Eq. 43).

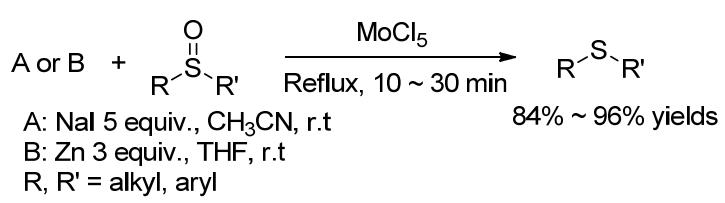

2008 年, Shockravi 等 ${ }^{[56]}$ 利用 $\mathrm{Zn} / \mathrm{AcOH} / \mathrm{SiO}_{2}$ 体系在 微波辐射的作用下还原了一系列的亚砜，该方法的反应 速率极快 $(40 \mathrm{~s}$ 以内), 并且收率和化学选择性都不错 (Eq. 44).

$$
\begin{aligned}
& \mathrm{Zn}+\underset{\mathrm{R}^{-\mathrm{S}_{-}}}{\stackrel{\mathrm{O}}{\mathrm{R}^{\prime}}} \stackrel{\mathrm{AcOH}, \mathrm{SiO}_{2}}{\mathrm{MW}, 40 \mathrm{~s}} \mathrm{R}^{-\mathrm{S}_{\mathrm{CR}^{\prime}}} \\
& \mathrm{R}, \mathrm{R}^{\prime}=\text { alkyl, benzyl, aryl } \quad 87 \% \sim 93 \% \text { yields }
\end{aligned}
$$

2009 年, $\mathrm{Oh}$ 和 $\mathrm{Knabe}^{[57]}$ 利用 $\mathrm{NbCl}_{5}$ 和锌粉在四氢呋 喃与苯混合溶剂中原位生成的低价铌配合物还原了相 应的亚砜，该反应的反应条件温和且操作简单，同时化 学选择性和产率都很高, 并且该方法不仅能还原亚砜, 还能还原环氧化物以及一些胺- $N$-氧化物(Eq. 45). 


$$
\begin{aligned}
& \mathrm{R}^{\stackrel{\mathrm{O}}{\mathrm{S}}{ }_{-\mathrm{R}^{\prime}}} \frac{\mathrm{Zn}, \mathrm{NbCl}_{5}}{\mathrm{THF} / \mathrm{PhH}, 23^{\circ} \mathrm{C}, 0.5 \sim 24 \mathrm{~h}} \mathrm{R}^{-\mathrm{S}_{\mathrm{R}^{\prime}}} \\
& R, R^{\prime}=\text { benzyl, alkyl, phenyl } \\
& 78 \% \sim 99 \% \text { yields }
\end{aligned}
$$

2015 年, Yoo 等 ${ }^{[58]}$ 发现 $\mathrm{HfCl}_{4} / \mathrm{Zn}$ 体系也能够以高产 率还原一系列的亚砜, 在此之前已经有关于 $\mathrm{HfCl}_{4} /$ $\mathrm{KBH}_{4}$ 体系对亚砜还原的报道, 与使用 $\mathrm{HfCl}_{4} / \mathrm{KBH}_{4}$ 体系 还原亚砜的方法相比, $\mathrm{HfCl}_{4} / \mathrm{Zn}$ 体系在相对较短的时间 内为相同的底物提供了更好的收率. 该体系在室温下就 能还原各类芳香族和脂肪族亚砜, 且对官能团具有高的 化学选择性, 碳碳双键、硝基、氰基、醛基等在还原过 程中不受影响(Eq. 46).

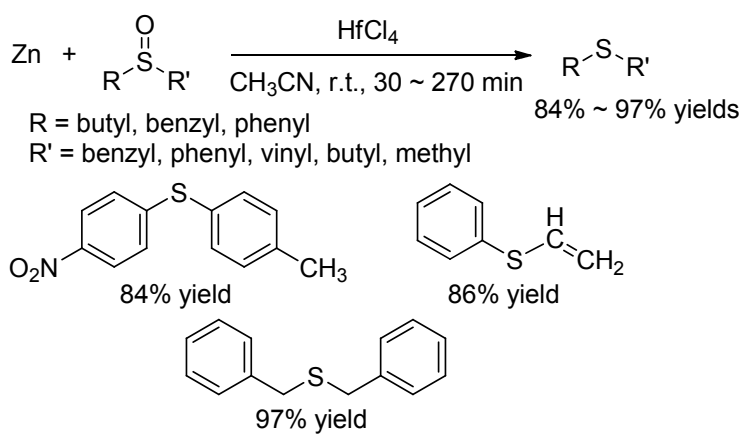

2007 年时, 铟金属由于其独特的性质(水和空气中 的低毒性以及高稳定性) 而受到越来越多的关注. Yoo 等 ${ }^{[59]}$ 研究了 $\mathrm{MoCl}_{5} / \mathrm{In}$ 体系对各类亚砜的还原(Eq. 47), 在氮气环境下将铟粉加入到五氯化钼的四氢呋喃溶液 中便可得到该还原体系，该体系的反应时间很短 $(5$ $\min$ ), 能够以高产率迅速将亚砜还原为相应的硫醚, 并 且对官能团的化学选择性良好, 乙烯基和醛基等在还原 过程中不受影响。

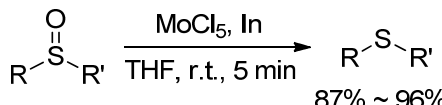

$\mathrm{R}, \mathrm{R}^{\prime}$ = phenyl, alkyl, benzyl<smiles></smiles><smiles>C=CSc1ccccc1</smiles>

同年 Yoo 小组 ${ }^{[60]}$ 又利用 $\mathrm{WCl}_{6} / \mathrm{In}$ 的还原体系对二烷 基亚砜、二芳基亚砜和芳基烷基亚砜进行了还原，该方 法的优势在于其较快的反应速率, 较高的产率以及良好 的化学选择性(Eq. 48).

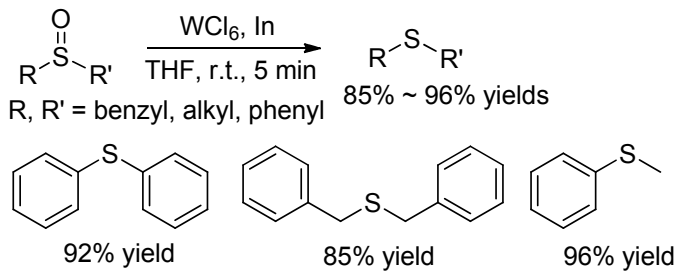

2013 年, Yoo 等 ${ }^{[61]}$ 通过在超声处理下将铟粉加入到 五氯化铌的四氢呋喃溶液中, 得到了 $\mathrm{NbCl}_{5} / \mathrm{In}$ 的还原体 系，该体系的反应条件温和，步骤简单。作者们还尝试 利用此体系还原二苯砜和甲基苯砜，但遗憾的是反应并 没有发生(Eq. 49).

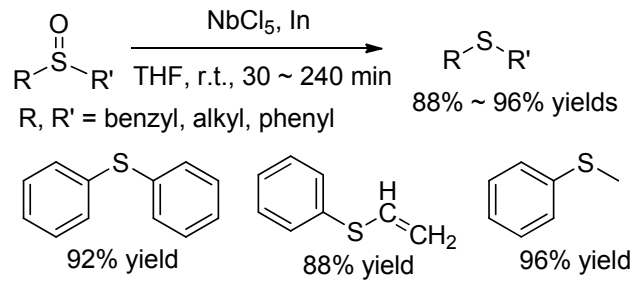

2016 年, Yoo 研究小组 ${ }^{[62]}$ 发现铟粉和 $\mathrm{TaCl}_{5}$ 组成的 体系也能够对亚砜进行还原。该方法的反应条件温和 (乙腈中室温反应)且硫醚收率较高，可以容忍一系列官 能团，如卤素基团、甲氧基、醛基、碳碳双键等(Eq. 50).

$$
\begin{aligned}
& \mathrm{R}=\text { phenyl, butyl, benzyl } \\
& \mathrm{R}^{\prime}=\text { phenyl, benzyl, methyl, vinyl }
\end{aligned}
$$
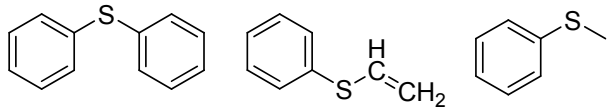

除了上述例子以外，其他过渡金属也应用到亚砜的 还原反应中, 如 2002 年 Yoo 等 ${ }^{[63]}$ 利用 $\mathrm{Cp}_{2} \mathrm{TiCl}_{2} / \mathrm{In}$ 体系 在温和条件下以良好的收率将各种亚砜还原为相应的 硫化物. 该体系以 THF 作为溶剂, 室温下反应(Eq. 51).

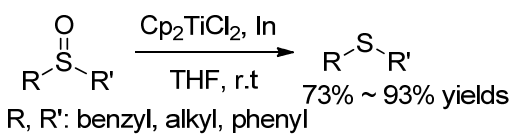

2010 年, Zhang 等 ${ }^{[64]}$ 则利用 $\mathrm{Cp}_{2} \mathrm{TiCI}_{2} / \mathrm{Al}$ 体系对各类 亚砜进行了还原. 在该反应中，亚砜中的 $\mathrm{S}-\mathrm{O}$ 键被裂 解并且氧原子被转移到 $\mathrm{Cp}_{2} \mathrm{TiCl}_{2}$ 以产生稳定的 +4 价钛 络合物，从而生成产物硫醚(Eq. 52).

$$
\underset{\mathrm{R}^{-} \mathrm{S}_{-\mathrm{R}^{\prime}}}{\mathrm{O}} \stackrel{\mathrm{Cp}_{2} \mathrm{TiCl}_{2}, \mathrm{Al}}{\mathrm{THF}} \mathrm{R}^{-\mathrm{S}_{\mathrm{R}^{\prime}}}
$$

金属一金属盐的二元体系，例如 $\mathrm{Al}-\mathrm{SbCl}_{3} 、 \mathrm{Zn}-\mathrm{SbCl}_{3}$ 、 Al- $\mathrm{NiCl}_{2} \cdot 6 \mathrm{H}_{2} \mathrm{O}-\mathrm{THF}$ 等，长期被用作许多官能团的还原 剂, 2005 年, Saikia 等 ${ }^{[65]}$ 利用 $\mathrm{Al} / \mathrm{NiCl}_{2} \cdot 6 \mathrm{H}_{2} \mathrm{O}$ 体系在室温 下以 $\mathrm{THF}$ 为溶剂还原了一系列烷基芳基和二烷基亚砜, 该方法反应条件温和, 试剂成本低且产物分离方便(Eq. $53)$. 


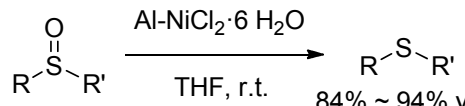

$R, R^{\prime}=$ alkyl, aryl<smiles>CSc1ccccc1OCCCOc1ccccc1</smiles>

2007 年, Khurana 等 ${ }^{[66]}$ 利用 $\mathrm{Mg} / \mathrm{MeOH}$ 的还原体系 对硒氧化物、碲氧化物、砜和亚砜等物质进行了还原(Eq. 54), 通常需要加入过量的 $\mathrm{Mg}$ 以完成反应. 该方法的优 势在于操作简单, 反应条件温和(室温下)以及对环境友 好性.

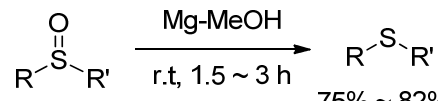

$$
\begin{aligned}
& \mathrm{R}, \mathrm{R}^{\prime}=\text { phenyl, benzyl } 75 \% \sim 82 \% \text { yields }
\end{aligned}
$$

2007 年, Yoo 等 ${ }^{[67]}$ 报道了利用 $\mathrm{Mo}(\mathrm{CO})_{6}$ 作为还原剂 还原亚砜的方法. 经过条件的耖选，作者发现二烷基、 二芳基和芳基烷基亚砜都能在温和的反应条件下于 2 $4 \mathrm{~h}$ 之内还原成相应的硫化物(Eq. 55), 反应收率较高.

$$
\begin{aligned}
& \mathrm{Mo}(\mathrm{CO})_{6}+\underset{\mathrm{R}^{-} \mathrm{S}_{-\mathrm{R}^{\prime}}}{\stackrel{\mathrm{O}}{\text { Reflux, } 2 \sim 4 \mathrm{~h}}} \stackrel{\text { EtOH }}{\longrightarrow} \mathrm{R}^{-\mathrm{S}_{{ }^{\prime}} \mathrm{R}^{\prime}} \\
& \mathrm{R}, \mathrm{R}^{\prime}=\text { alkyl, phenyl, benzyl } \quad 84 \% \sim 96 \% \text { yields }
\end{aligned}
$$

\section{7 醇类化合物还原亚砜}

醇类化合物作为还原剂可以用来还原亚砜. Swern 氧化反应, 利用草酰氯、二甲基亚砜和三乙胺可以将醇 氧化为相应的羰基化合物. 受此启发, Gurpreet 等 ${ }^{[68]}$ 在 2004 年以四氢呋喃作为溶剂, 在三乙胺的存在下利用 异丙醇和草酰氯对一系列含有 $\alpha$ 氢的亚砜进行了还原 (Eq. 56).

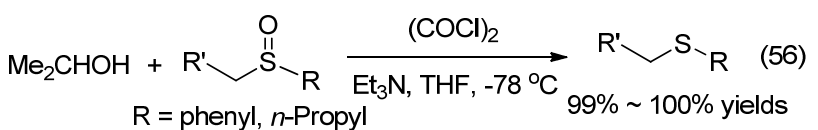

$$
\begin{aligned}
& \mathrm{R}^{\prime} \text { = phenyl, methyl }
\end{aligned}
$$

反应于 $-78{ }^{\circ} \mathrm{C}$ 下进行, 操作简单, 收率优秀, 并且 具有很好的官能团容忍性, 反应机理如 Scheme 12 所示.

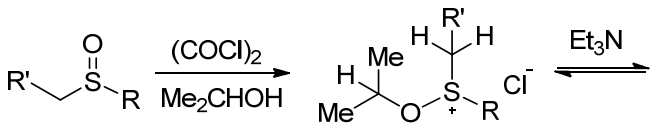

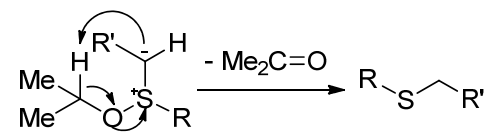

图式 $12 \mathrm{CoCl}_{2} / \mathrm{Me}_{2} \mathrm{CHOH}$ 还原亚砜的反应机理研究 Scheme 12 Proposed mechanism for the reduction of sulfoxides with $\mathrm{CoCl}_{2} / \mathrm{Me}_{2} \mathrm{CHOH}$
2012 年, Sanz 等 ${ }^{[69]}$ 报道了一种新的环境友好的亚砜 还原剂频哪醇, 在二氯二氧代钼配合物作为催化剂的情 况下，反应在极短的时间内(10 min 以内)以高收率地还 原了一系列亚砜(包括含有还原性官能团的亚砜)(Eq. $57)$, 丙酮和水是该反应的唯一副产物.

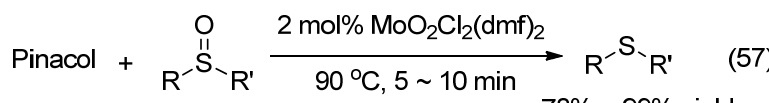

$$
\begin{aligned}
& \mathrm{R}=\text { phenyl, alkyl } \\
& \mathrm{R}^{\prime} \text { = phenyl, alkyl, allyl } \\
& \text { (18\% yield } \\
& \overbrace{91 \% \text { yield }}^{s}
\end{aligned}
$$

2013 年, Sanz 等 ${ }^{[70]}$ 发现甘油能够在二氧代钼配合物 作为催化剂的情况下对亚砜进行还原, 并且在使用粗甘 油的情况下反应仍然能够进行. 甘油与二氧代钼配合物 都是易得又环保的物质，使用这种廉价、绿色的反应体 系, 反应后很容易地将产物与催化剂分离, 从而进行再 循环利用. 作者在利用循环五次过后的体系对亚砜进行 还原时，依然能够以 $80 \%$ 以上的收率得到相应的硫醚产 物(Eq. 58).

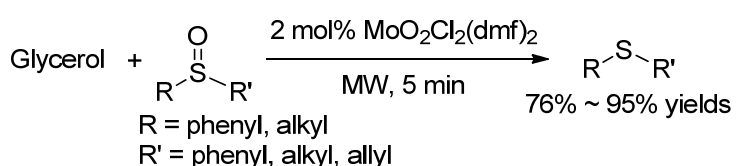

2016 年, Abbasi 等 ${ }^{[71]}$ 利用抗坏血酸在 $N$-溴代丁二酰 亚胺进行催化的情况下对一系列亚砜进行了还原，并以 较高的收率得到了还原后的产物硫醚(Eq. 59).

$$
L \text {-ascorbic acid }+\underset{R^{-S}}{\stackrel{O}{\|}} \stackrel{\text { NBS }}{\stackrel{\text { EtOAc, r.t }}{\longrightarrow}} R^{-S^{\prime}}{ }_{R^{\prime}}
$$

$$
R, R^{\prime}=\text { phenyl, benzyl, aryl } \quad 88 \% \sim 92 \% \text { yields }
$$

作者所使用的抗坏血酸是一种绿色环保以及便宜 易得的反应原料, 并且反应过后所生成的副产物，如去 氢抗坏血酸和琥珀酰亚胺等物质, 也不会对环境产生危 害. 另外该反应实验操作的简单性也使得该方法的实用 性大大增强, 反应的机理如 Scheme 13 所示.

\section{8 氢气还原亚砜}

利用氢气作为还原剂来还原亚砜引起了研究人员 的极大兴趣. 2014 年 Kaneda 研究小组 ${ }^{[72]}$ 发现钉纳米粒 子在氢气作为还原剂的情况下能够用来催化一系列亚 砜的还原. 钉纳米粒子的催化活性受载体材料的影响, 

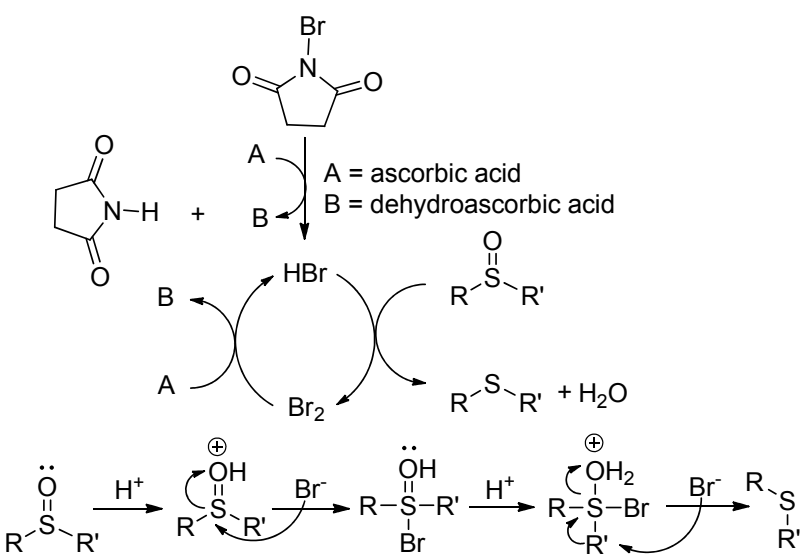

图式 13 NBS 和抗坏血酸还原亚砜反应机理研究

Scheme 13 A proposed mechanism with ascorbic acid and NBS

其中 $\mathrm{TiO}_{2}$ 作为载体时效率最高, 且随着粒径的减小, 钉 纳米粒子的催化活性逐渐增加. 作者最终在 $101 \mathrm{kPa}$ 的 氢气环境下用 $5 \mathrm{~mol} \%$ 的 $\mathrm{Ru} / \mathrm{TiO}_{2}$ 催化还原了各类亚砜, 收率优秀且催化剂易于回收, 耐用性强(Eq. 60).

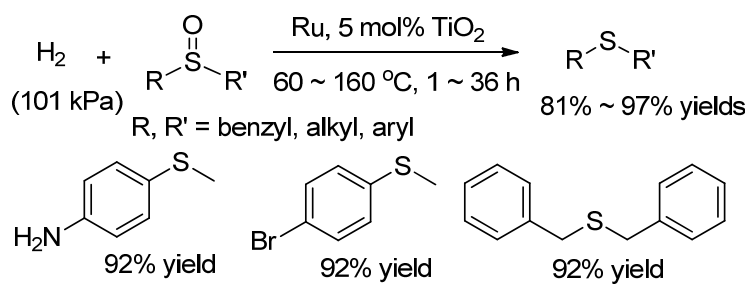

2016 年, Shimizu 等 ${ }^{[73]}$ 利用 $\mathrm{Pt}$ 和 $\mathrm{MoO}_{x}$ 共负载的 $\mathrm{TiO}_{2}$ 催化剂在无溶剂的条件下对亚砜进行了还原(Eq. $61)$, 其中氢气为反应的还原剂, 反应只需要 $0.1 \mathrm{~mol} \%$ 的催化剂用量就能在温和条件下 $\left(50 \sim 155{ }^{\circ} \mathrm{C}, 101\right.$ 或者 $707 \mathrm{kPa}$ 大气压力) 以高收率还原一系列的亚砜. $\mathrm{Pt}-\mathrm{MoO}_{x} / \mathrm{TiO}_{2}$ 显示出比先前报道的催化剂更高的转换 数, 并且是一种可重复使用的催化剂, 在循环使用五次 之后仅观察到轻微的活性损失. 此外该催化剂还能将茮 基苯基砜还原成茮基苯基硫醚，这是第一个在催化条件 下用氢气将砜还原成为硫醚的例子.

$$
\mathrm{H}_{2}+\mathrm{R}_{\mathrm{R}} \mathrm{R}_{\mathrm{S}}^{\mathrm{O}} \mathrm{R}^{\prime}
$$

$92 \%$ yield
$0.1 \mathrm{~mol} \% \mathrm{Pt}-\mathrm{MoO}_{x}, \mathrm{TiO}_{2}$

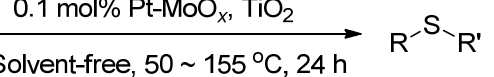
$81 \% \sim 97 \%$ yields<smiles>CSc1ccc(Br)cc1</smiles>

91\% yield

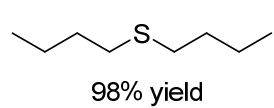

$98 \%$ yield
最近 Uematsu 等 ${ }^{[74]}$ 发现在非均相催化剂 $\mathrm{Pt} /$ $\mathrm{V}_{1-x} \mathrm{Cr}_{x}-\mathrm{Hol}$ 的催化作用下, 可以在 $101 \mathrm{kPa}$ 的氢气环境 下对各类烷基芳基亚砜进行催化还原。其中催化剂 $\mathrm{V}_{1-x} \mathrm{Cr}_{x}-\mathrm{Hol}$ 在催化还原亚砜之后 $\mathrm{V}^{3+}$ 被氧化为 $\mathrm{V}^{4+}$, 且 不能被氢气还原为 $\mathrm{V}^{3+}$, 而在铂负载的情况下, $\mathrm{V}^{4+}$ 则可
以与氢气作用还原为 $\mathrm{V}^{3+}$. 该方法的官能团容忍性较好, 亚砜能够选择性地还原为相应的硫醚, 并且该催化剂可 以重复用于亚砜脱氧，但性能会有所降低. 此外该催化 剂还能催化吡啶 $N$-氧化物等物质的还原(Eq. 62).

$$
\text { 等 }
$$

\section{9 其他试剂还原亚砜}

最后我们将介绍一些其他试剂还原亚砜的方法. 如 2004 年 Boyd 等 ${ }^{[75]}$ 在立体选择性还原酶催化下使用好氧 菌和厌氧菌还原了一系列亚砜，并且研究小组人员收集 了一系列的酶催化该反应的证据.

2005 年, Nakayama 等 ${ }^{[76]}$ 发现四氰基环氧乙烷 (TCNEO)不仅能够用来进行硫醚的氧化, 还能够用来进 行亚砜的还原，反应在室温下便能进行，不过反应速率 较慢, 收率中等.

2001 年, Jenks 等 ${ }^{[77]}$ 在醇盐存在的情况下于甲醇溶 剂中对芳香族亚砜进行光解，从而得到了相应的芳香族 硫醚. 2015 年 Suzuki 等 ${ }^{[78]}$ 也在可见光催化的情况下. 利 用 $\mathrm{TBA}_{4} \mathrm{H}_{4}\left[\gamma-\mathrm{SiW}_{10} \mathrm{O}_{36}\right]\left(\mathrm{SiW}_{10}\right)$ 还原了一系列亚砜.

2012 年, Fernandes 等 ${ }^{[79]}$ 提出了一个新颖的不需要 加入还原剂来还原亚砜的方案, 该方法只需要以氯仿为 溶剂，在回流的条件下用 $10 \mathrm{~mol} \%$ 的 $\mathrm{ReOCl}_{3}\left(\mathrm{PPh}_{3}\right)_{2}$ 来催 化反应的进行. 这样不仅节省了大量的还原剂，使反应 更加的简单实用，而且对环境更加友好. 此外中等到优 秀的收率, 较好的化学选择性以及底物的普适性也增加 了该方法的可行性(Eq. 63).

$$
\begin{aligned}
& \mathrm{R}^{-1} \stackrel{\mathrm{O}}{\mathrm{S}}{ }_{-\mathrm{R}^{\prime}} \frac{\mathrm{ReOCl}_{3}\left(\mathrm{PPh}_{3}\right)_{2}}{\mathrm{CHCl}_{3} \text {, reflux, } 17 \sim 21 \mathrm{~h}} \mathrm{R}^{-\mathrm{S}_{-\mathrm{R}^{\prime}}} \\
& \mathrm{R}=\text { benzyl, alkyl, aryl } \quad 70 \% \sim 98 \% \text { yields } \\
& \mathrm{R}^{\prime}=\text { aryl, benzyl, alkyl, alkyl acetate }
\end{aligned}
$$

2014 年, Kominami 等 ${ }^{[80}$ 报道了在二氧化钛颗粒的 乙腈悬浮液中利用微波辐射对亚砜进行脱氧, 反应在室 温下进行且不需要使用金属助催化剂和其他有毒试剂, 反应收率很高且时间很短 $(30 \mathrm{~min}$ 以内).

\section{0 结论与展望}

硫醚化合物是有机化学中一类重要的有机化合物 与有机合成中间体，易于通过亚砜的还原反应进行制 备. 本文归纳了近年来含磷化合物、含硼化合物、含硫 化合物、含硅化合物、卤化物、金属及其化合物、醇类 化合物以及氢气和其他杂项试剂还原亚砜的方法. 这些 
由各个研究小组所发展的方法大多实用而有效, 并且随 着金属有机的发展, 很多研究人员都选择在体系中加入 金属及其配合物作为催化剂来加速反应的进行. 相比于 早些年的亚砜还原体系, 这些方法在实用性、高效性以 及环保性等方面的进步是显而易见的, 但尽管如此, 它
们的不足之处也是客观存在的. 亚砜的还原反应仍然是 有机反应当中的一个挑战性课题，仍有许多研究小组致 力于开发新的亚砜还原体系，并且毫无疑问，开发更加 环保、便捷、高效的还原体系是所有研究人员的研究目 标. 在此对本文所涉及的还原方法进行了总结(表 1).

表 1 亚砜还原方法总结

Table 1 Summary of sulfoxide reduction methods

\begin{tabular}{|c|c|c|c|}
\hline & 还原体系 & 收率范围 & 参考文献 \\
\hline \multirow{10}{*}{ 含磷化合物 } & $\mathrm{TCEP} / \mathrm{I}_{2}$ & $72 \% \sim 99 \%$ & Ref. [12] \\
\hline & $\mathrm{P}(\mathrm{OPh})_{3} / \mathrm{MoO}_{2} \mathrm{Cl}_{2}(\mathrm{dmf})_{2}$ & $52 \% \sim 98 \%$ & Ref. [13] \\
\hline & $\mathrm{PPh}_{3} / \mathrm{TiCl}_{4}$ & $67 \% \sim 97 \%$ & Ref. [14] \\
\hline & {$\left[\mathrm{PCl}_{3-n}\left(\mathrm{SiO}_{2}\right)_{n}\right] / \mathrm{I}_{2}$} & $90 \% \sim 100 \%$ & Ref. [15] \\
\hline & Woollins 试剂 & $81 \% \sim 99 \%$ & Ref. [16] \\
\hline & $\mathrm{PPh}_{3} / \mathrm{Br}_{2} / \mathrm{CuBr}$ & $90 \% \sim 98 \%$ & Ref. [17] \\
\hline & $\mathrm{PPh}_{3} /[\mathrm{RMIM}]_{2}\left[\mathrm{Mo}(\mathrm{O})_{2}(\mathrm{NCS})_{4}\right]$ & $83 \% \sim 99 \%$ & Ref. [18] \\
\hline & $\mathrm{PPh}_{3} / \mathrm{SOCl}_{2}$ & $92 \% \sim 99 \%$ & Ref. [19] \\
\hline & $\mathrm{PPh}_{3} / \mathrm{TBBDA}$ & $91 \% \sim 98 \%$ & Ref. [20] \\
\hline & $\mathrm{PCl}_{3}$ & $66 \% \sim 99 \%$ & Ref. [21] \\
\hline \multirow{10}{*}{ 含硼化合物 } & $\mathrm{NaBH}_{4} / \mathrm{I}_{2}$ & $57 \% \sim 98 \%$ & Ref. [22] \\
\hline & HBcat & $78 \% \sim 95 \%$ & Ref. [23] \\
\hline & $\mathrm{HBBr}_{2} / \mathrm{Me}_{2} \mathrm{~S}$ & $90 \% \sim 92 \%$ & Ref. [24] \\
\hline & $\mathrm{HBcat} / \mathrm{MoO}_{2} \mathrm{Cl}_{2}\left(\mathrm{H}_{2} \mathrm{O}\right)_{2}$ & $85 \% \sim 95 \%$ & Ref. [25] \\
\hline & $\mathrm{BH}_{3}-\mathrm{THF} / \mathrm{MoO}_{2} \mathrm{Cl}_{2}$ & $85 \% \sim 93 \%$ & Ref. [25] \\
\hline & $\mathrm{HfCl}_{4} / \mathrm{KBH}_{4}$ & $71 \% \sim 100 \%$ & Ref. [26] \\
\hline & $\mathrm{HBcat} / \mathrm{ReIO}_{2}\left(\mathrm{PPh}_{3}\right)_{2}$ & $90 \% \sim 95 \%$ & Ref. [27] \\
\hline & $\mathrm{NaBH}_{4} /$ moist alumina & $12 \% \sim 99 \%$ & Ref. [28] \\
\hline & $\mathrm{HBcat} / \mathrm{Zn}(\mathrm{OTf})_{2}$ & $85 \% \sim 99 \%$ & Ref. [29] \\
\hline & $\mathrm{B}(\mathrm{Pin})_{2} / \mathrm{Zn}(\mathrm{OTf})_{2}$ & $83 \% \sim 98 \%$ & Ref. [30] \\
\hline \multirow{10}{*}{ 卤化物 } & $\mathrm{TiI}_{4}$ & $80 \% \sim 97 \%$ & Ref. [31] \\
\hline & $\mathrm{NaI} / \mathrm{ZrCl}_{4} 、 \mathrm{ZrOCl}_{2} \bullet 8 \mathrm{H}_{2} \mathrm{O}$ & $70 \% \sim 96 \%$ & Ref. [32] \\
\hline & $\mathrm{KI} / \mathrm{AcCl}$ & $87 \% \sim 95 \%$ & Ref. [33] \\
\hline & $\mathrm{KI} / \mathrm{Tf}_{2} \mathrm{O}$ & $91 \% \sim 98 \%$ & Ref. [34] \\
\hline & $\mathrm{KI} / \mathrm{TPAC}$ & $91 \% \sim 98 \%$ & Ref. [35] \\
\hline & $\mathrm{TCT} / \mathrm{H}_{2} \mathrm{O}_{2}$ & $93 \% \sim 99 \%$ & Ref. [36] \\
\hline & $\mathrm{NaI} /$ Silica sulfuric acid & $80 \% \sim 96 \%$ & Ref. [37] \\
\hline & $\mathrm{NaI} / \mathrm{CSA}$ & - & Ref. [38] \\
\hline & Silica bromide & $89 \% \sim 98 \%$ & Ref. [39] \\
\hline & $\mathrm{TaCl}_{5} / \mathrm{NaI}$ & $89 \% \sim 96 \%$ & Ref. [40] \\
\hline \multirow{5}{*}{ 含硫化合物 } & 3-巯基丙酸/ $\mathrm{I}_{2}(\mathrm{NBS})$ & $55 \% \sim 98 \%$ & Ref. [41] \\
\hline & 3-巯基丙酸/TMCS & $40 \% \sim 97 \%$ & Ref. [42] \\
\hline & 硫代乙酸/I & $75 \% \sim 98 \%$ & Ref. [43] \\
\hline & $\mathrm{NaHSO}_{3} / \mathrm{I}_{2}$ & $85 \% \sim 96 \%$ & Ref. [44] \\
\hline & $\mathrm{MPS} / \mathrm{MoO}_{2} \mathrm{Cl}_{2}(\mathrm{dmf})_{2}$ & $67 \% \sim 97 \%$ & Ref. [45] \\
\hline \multirow{9}{*}{ 含硅化合物 } & $\mathrm{PhSiH}_{3} / \mathrm{MoO}_{2} \mathrm{Cl}_{2}$ & $92 \% \sim 97 \%$ & Ref. [46] \\
\hline & $\mathrm{PhSiH}_{3} / \mathrm{ReIO}_{2}\left(\mathrm{PPh}_{3}\right)_{2}$ & $80 \% \sim 100 \%$ & Ref. [47] \\
\hline & $\mathrm{PhSiH}_{3}(\mathrm{HBcat}) / \mathrm{HReO}_{4}$ & $55 \% \sim 98 \%$ & Ref. [48] \\
\hline & $\mathrm{PhSiH}_{3} / \mathrm{Zn}(\mathrm{OTf})_{2}$ & $69 \% \sim 99 \%$ & Ref. [49] \\
\hline & $\mathrm{Ph}_{2} \mathrm{SiH}_{2} / \mathrm{Cu}(\mathrm{acac})_{2}$ & $62 \% \sim 99 \%$ & Ref. [50] \\
\hline & $\mathrm{PhSiH}_{3}(\mathrm{PMHS}) /\left[\mathrm{Fe}_{2}(\mathrm{Co})_{9}\right]$ & - & Ref. [51] \\
\hline & $\mathrm{PhSiH}_{3} / \mathrm{ReOBr}_{2}($ hmpbta $)\left(\mathrm{PPh}_{3}\right)$ & $76 \% \sim 95 \%$ & Ref. [52] \\
\hline & $\mathrm{PhSiH}_{3} / \mathrm{B}\left(\mathrm{C}_{6} \mathrm{~F}_{5}\right)_{3}$ & $60 \% \sim 87 \%$ & Ref. [53] \\
\hline & $\mathrm{Et}_{3} \mathrm{SiH} / \mathrm{B}\left(\mathrm{C}_{6} \mathrm{~F}_{5}\right)_{3}$ & $48 \% \sim 90 \%$ & Ref. [54] \\
\hline
\end{tabular}




\begin{tabular}{|c|c|c|c|}
\hline & & & 续表 \\
\hline & 还原体系 & 收率范围 & 参考文献 \\
\hline \multirow{13}{*}{ 金属及其化合物 } & $\mathrm{Zn}(\mathrm{NaI}) / \mathrm{MoCl}_{5}$ & $84 \% \sim 96 \%$ & Ref. [55] \\
\hline & $\mathrm{Zn} / \mathrm{AcOH} / \mathrm{SiO}_{2}$ & $87 \% \sim 93 \%$ & Ref. [56] \\
\hline & $\mathrm{NbCl}_{5} / \mathrm{Zn}$ & $78 \% \sim 99 \%$ & Ref. [57] \\
\hline & $\mathrm{HfCl}_{4} / \mathrm{Zn}$ & $84 \% \sim 97 \%$ & Ref. [58] \\
\hline & $\mathrm{MoCl}_{5} / \mathrm{In}$ & $87 \% \sim 96 \%$ & Ref. [59] \\
\hline & $\mathrm{WCl}_{6} / \mathrm{In}$ & $85 \% \sim 96 \%$ & Ref. [60] \\
\hline & $\mathrm{NbCl}_{5} / \mathrm{In}$ & $88 \% \sim 96 \%$ & Ref. [61] \\
\hline & $\mathrm{TaCl}_{5} / \mathrm{In}$ & $87 \% \sim 95 \%$ & Ref. [62] \\
\hline & $\mathrm{Cp}_{2} \mathrm{TiCl}_{2} / \mathrm{In}$ & $73 \% \sim 93 \%$ & Ref. [63] \\
\hline & $\mathrm{Cp}_{2} \mathrm{TiCI}_{2} / \mathrm{Al}$ & - & Ref. [64] \\
\hline & $\mathrm{Al} / \mathrm{NiCl}_{2} \cdot 6 \mathrm{H}_{2} \mathrm{O}$ & $84 \% \sim 94 \%$ & Ref. [65] \\
\hline & $\mathrm{Mg} / \mathrm{MeOH}$ & $75 \% \sim 82 \%$ & Ref. [66] \\
\hline & $\mathrm{Mo}(\mathrm{CO})_{6}$ & $84 \% \sim 96 \%$ & Ref. [67] \\
\hline \multirow{4}{*}{ 醇类化合物 } & 异丙醇/草酰氯 & $99 \% \sim 100 \%$ & Ref. [68] \\
\hline & 频哪醇 $/ \mathrm{MoO}_{2} \mathrm{Cl}_{2}(\mathrm{dmf})_{2}$ & $78 \% \sim 99 \%$ & Ref. [69] \\
\hline & 甘油/ $\mathrm{MoO}_{2} \mathrm{Cl}_{2}(\mathrm{dmf})_{2}$ & $76 \% \sim 95 \%$ & Ref. [70] \\
\hline & 抗坏血酸/NBS & $88 \% \sim 92 \%$ & Ref. [71] \\
\hline \multirow{3}{*}{ 氢气 } & $\mathrm{H}_{2} / \mathrm{Ru} / \mathrm{TiO}_{2}$ & $81 \% \sim 97 \%$ & Ref. [72] \\
\hline & $\mathrm{H}_{2} / \mathrm{Pt}-\mathrm{MoO}_{x} / \mathrm{TiO}_{2}$ & $81 \% \sim 97 \%$ & Ref. [73] \\
\hline & $\mathrm{H}_{2} / \mathrm{Pt} / \mathrm{V}_{(1-x)} \mathrm{Cr}_{x}-\mathrm{Hol}$ & $82 \% \sim 98 \%$ & Ref. [74] \\
\hline \multirow{6}{*}{ 其他杂项试剂 } & 好氧菌和厌氧菌 & - & Ref. [75] \\
\hline & 四氰基环氧乙烷 & - & Ref. [76] \\
\hline & 光解 & $87 \% \sim 95 \%$ & Ref. [77] \\
\hline & $\mathrm{TBA}_{4} \mathrm{H}_{4}\left[\gamma-\mathrm{SiW}_{10} \mathrm{O}_{36}\right]\left(\mathrm{SiW}_{10}\right)$ & $91 \% \sim 98 \%$ & Ref. [78] \\
\hline & $\mathrm{ReOCl}_{3}\left(\mathrm{PPh}_{3}\right)_{2}$ & $91 \% \sim 98 \%$ & Ref. [79] \\
\hline & $\mathrm{TiO}_{2} /$ microwave & $93 \% \sim 99 \%$ & Ref. [80] \\
\hline
\end{tabular}

\section{References}

[1] Chen, C.; Li, Y.; Xiao, Y.; Zhu, L.; Cheng, C.; Liu, Y. Chin. J. Org. Chem. 2011, 31, 925 (in Chinese). (陈春玉, 李毅, 肖英, 朱林, 程长明, 刘杨, 有机化学, 2011, 31, 925.)

[2] (a) McGarrigle, E. M.; Myers, E. L.; Illa, O.; Shaw, M. A.; Riches, S. L.; Aggarwal, V. K. Chem. Rev. 2007, 107, 5841.

(b) Rickard, D.; Luther III, G. W. Chem. Rev. 2007, 107, 514.

[3] Michaelis, A.; Godchaux, E. Ber. 1891, 24, 757.

[4] Loth, F.; Michaelis, A. Ber. 1894, 27, 2540.

[5] Madesclaire, M. Tetrahedron Lett. 1988, 44, 6537.

[6] Still, N. W. J. In Comprehensive Organic Synthesis, Vol. 8, Ed.: Trost, B. M., Pergamon Press, Oxford, 1991, p. 403.

[7] Kukushkin, V. Y. Coord. Chem. Rev. 1995, 139, 375.

[8] Firouzabadia, H.; Jamalianb, A. J. Sulfur Chem. 2008, 29, 53.

[9] Gaumont, A. C.; Gulea, M. S.; Reboul, V. Comprehensive Organic Synthesis II, 2nd ed., Vol. 8, Elsevier, Amsterdam, 2014, p. 535.

[10] Shiri, L.; Kazemi, M. Res. Chem. Intermed. 2017, 43, 6007.

[11] Wakisaka, M.; Hatanaka, M.; Nitta, H.; Hatamura, M.; Ishizuka,T. Synthesis. 1980, 67.

[12] Faucher, A.; Grand-Maitre, C. Synth. Commun. 2003, 33, 3503.

[13] Sanz, R.; Escribano, J.; Aguado, R.; Pedrosa, M. R.; Arnáiz, F. J. Synthesis 2004, 35, 1629.

[14] Kikuchi, S.; Konishi, H.; Hashimoto, Y. Tetrahedron Lett. 2005, 61, 3587.

[15] Iranpoor, N.; Firouzabadi, H.; Jamalian, A. Synlett 2005, 36, 1447.

[16] Hua, G.; Woollins, J. D. Tetrahedron Lett. 2007, 48, 3677.

[17] Bahrami, K.; Khodaei, M. M.; Khedri, M. Chem. Lett. 2007, 36, 1324.

[18] Bagherzadeh, M.; Ghazali-Esfahan, S. New J. Chem. 2012, 36, 971.

[19] Jang, Y.; Kim, K. T.; Jeon, H. B. J. Org. Chem. 2013, 78, 6328.
[20] Ghorbani-Vaghei, R.; Shiri, L.; Ghorbani-Choghamarani, A. C. R. Chim. 2014, 17, 1002

[21] Zhao, X.; Zheng, X.; Yang, B.; Sheng, J.; Lu, K. Org. Biomol. Chem. 2018, 16, 1200.

[22] Karimi, B.; Zareyee, D. Synthesis 2003, 34, 335.

[23] Harrison, D. J.; Tam, N. C.; Vogels, C. M.; Langler, R. F.; Baker, R. T.; Deckenc, A.; Westcott, S. A. Tetrahedron Lett. 2004, 45, 8493.

[24] Chandra, R. D.; Herbert, B. C. J. Chem. Res. 2006, 10, 642.

[25] Fernandes, A. C.; Carlos, C. R. Tetrahedron Lett. 2007, 48, 9176.

[26] Zhang, J.; Gao, X.; Zhang, C.; Luan, J.; Zhao, D. Synth. Commun. 2010, 40, 1794.

[27] Fernandes, A. C.; Fernandes, J. A.; Romao, C. C.; Veiros, L. F.; Calhorda, M. J. Organometallics 2010, 29, 5517.

[28] Yakabe, S.; Morimoto, M. T. Synth. Commun. 2011, 41, 2251.

[29] Enthaler, S.; Krackl, S.; Irran, E.; Inoue, S. Catal. Lett. 2012, 142, 1003.

[30] Enthaler, S. Catal. Lett. 2012, 142, 1306.

[31] Shimizu, M.; Shibuya, K.; Hayakawa, R. Synlett 2000, 10, 1437.

[32] Firouzabadi, H.; Iranpoor, N.; Jafapour, M. J. Sulf. Chem. 2005, 26, 313.

[33] Ternois, J.; Guillen, F.; Piacenza, G.; Rose, S. J.; Plaquevent, C.; Coquerel, G. Org. Proc. Res. Develop. 2008, 12, 614.

[34] Bahrami, K.; Khodaei, M. M.; Karimi, A. Synthesis 2008, 16, 2543.

[35] Bahrami, K.; Khodaei, M. M.; Sheikh, A. M. J. Org. Chem. 2010, $75,6208$.

[36] Bahrami, K.; Khodaei, M. M.; Sohrabnezhad, S. Tetrahedron Lett. 2011, 52, 6420 .

[37] Zarei, M.; Ameri, A. M.; Jamalian, A. J. Sulfur Chem. 2013, 34, 259.

[38] Atabaki, F.; Abedini, E.; Shokrolahi, A. Phosphorus Sulfur Silicon Relat. Elem. 2015, 190, 1169

[39] Mohanazadeh, F.; Veisi, H.; Sedrpoushan, A.; Zolfigol, M. A.; Golmohammad, F.; Hemmati, S.; Hashemie, M. J. Sulfur Chem. 
2014, 35, 7 .

[40] Yoo, B. W.; Park, J.; Shin, H. J.; Yoon, C. M. J. Sulfur Chem. 2017, $38,597$.

[41] Karimi, B.; Zareyee, D. Synthesis 2003, 1875.

[42] Karimi, B.; Zareyee, D. Synthesis 2003, 335.

[43] Jabbari, A.; Zarei, M.; Jamaleddini, A. J. Sulfur Chem. 2012, 33, 413.

[44] Abbasi, M.; Mohammadizadeh, M. R.; Moradi, Z. Tetrahedron Lett. 2015, 56, 6610 .

[45] Garcia, N.; Fernandez-Rodriguez, M. A.; Garcia-Garcia, P.; Pedrosa, M. R.; Arnaiz, F. J.; Sanz, R. RSC Adv. 2016, 6, 27083.

[46] Fernandes, A. C.; Romao, C. C. Tetrahedron 2006, 62, 9650.

[47] Sousa, S. C. A.; Fernandes, A. C. Tetrahedron Lett. 2009, 50, 6872.

[48] Cabrita, S. I.; Sousa, C. A.; Fernandes, A. C. Tetrahedron Lett. 2010, 51,6132 .

[49] Enthaler, S. A. Catal. Sci. Technol. 2011, 1, 104.

[50] Enthaler, S.; Weidauer, M. Catal. Lett. 2011, 141, 833.

[51] Enthaler, S. Chem. Cat. Chem. 2011, 3, 666.

[52] Sousa, S. C. A.; Bernardo, J. R.; Wolff, M.; Machura, B.; Fernandes, A. C. Eur. J. Org. Chem. 2014, 46, 1855.

[53] Ding, F.; Jiang, Y.; Gan, S.; Bao, R. L. Y.; Lin, K.; Shi, L. Eur. J. Org. Chem. 2017, 24, 3427 .

[54] Porwal, D.; Oestreich, M. Synthesis 2017, 49, 4698.

[55] Firouzabadi, H.; Jamalian, A. Phosphorus Sulfur Relat. Elem. 2001, 170, 211.

[56] Shockravi, A.; Rostami, E.; Heidaryan, D.; Fattahi, H. Iran. J. Chem. Chem. Eng. 2008, 27, 129.

[57] Oh, K.; Knabe, W. E. Tetrahedron Lett. 2009, 65, 2966.

[58] Yoo, B. W.; Yu, B. R.; Yoon, C. M. J. Sulfur Chem. 2015, 36, 358.

[59] Woo, Y.; Byung, S.; Min, S.; Chol, P. M. Synth. Commun. 2007, 37, 3089.

[60] Yoo, B. W.; Park, M. C.; Song, M. S. Synth. Commun. 2007, 37, 4079.

[61] Yoo, B. W.; Kim, H. M.; Kim, D. Synth. Commun. 2013, 43, 2057.

[62] Yoo, B. W.; Lee, M. K.; Yoon, C. M. Phosphorus Sulfur Silicon Relat. Elem. 2016, 191, 807.

[63] Yoo, B. W.; Choi, K. H.; Kim, D. Y.; Choi, K. I.; Kim, J. H. Synth. Commun. 2003, 33, 53.
[64] Zhang, J.; Gao, X.; Zhang, C.; Luan, J.; Zhao, D. Synth. Commun. 2010, 40, 1794.

[65] Raju, B. R.; Devi, G.; Nongpluh, Y. S.; Saikia, A. K. Synlett 2005, 358.

[66] Khurana, J. M.; Sharma, V. S.; Chacko, A. Tetrahedron Lett. 2007, 63, 966.

[67] Yoo, B. W.; Song, M. S.; Park, M. C. Bull. Korean Chem. Soc. 2007, 28, 171.

[68] Gurpreet, S.; Bhatia, G. S.; Graczyk, P. P. Tetrahedron Lett. 2004, $45,5193$.

[69] Garcia, N.; Garcia-Garcia, P.; Fernandez-Rodriguez, M. A.; Rubio, R.; Pedrosa, M. R.; Arnaiz, F. J.; Sanz, R. Adv. Synth. Catal. 2012 $354,321$.

[70] Garcia, N.; Garcia-Garcia, P.; Fernandez-Rodriguez, M. A.; Rubio, R. M.; Pedrosa, R.; Arnaiz, F. J.; Sanz, R. Green Chem. 2013, 15, 999.

[71] Abbasi, M.; Mohammadizadeh, M. R.; Moradi, Z. Bull. Chem. Soc. Jpn. 2016, 89, 405.

[72] Mitsudome, T.; Takahashi, Y.; Mizugaki, T.; Jitsukawa, K.; Kaneda, K. Angew. Chem. 2014, 126, 8488

[73] Touchy, A. S.; Hakim-Siddiki, S. M. A.; Onodera, W.; Kon, K.; Shimizu, K. Green Chem. 2016, 18, 2554.

[74] Uematsu, T.; Miyamoto, Y.; Ogasawara, Y.; Suzuki, K.; Yamaguchi, K.; Mizuno, N. Catal. Sci. Technol. 2017, 7, 1912.

[75] Sharma, D. R.; Alistair, N. D.; King, W. T.; Shepherd, S. D.; Christopher, C. R.; Allen, R. A.; Holt, H. R. L.; Howard, D. Org. Biomol. Chem. 2004, 2, 554.

[76] Nakayama, J.; Tai, A.; Iwasa, S.; Furuya, T.; Sugihara, Y. Tetrahedron Lett. 2005, 46, 1395.

[77] Cubbage, J. W.; Tetzlaff, T. A.; Groundwater, H.; McCulla, R. D.; Nag, M.; Jenks, W. S. J. Org. Chem. 2001, 66, 8621.

[78] Suzuki, K.; Jeong, J.; Yamaguchi, K.; Mizuno, N. New J. Chem. 2015, 47, 1014.

[79] Sousa, S. C. A.; Bernardo, J. R.; Romao, C. C.; Fernandes, A. C. Tetrahedron Lett. 2012, 68, 8194 .

[80] Kominami, H.; Nakanishi, K.; Yamamoto, S.; Imamura, K.; Hashimoto, K. Catal. Commun. 2014, 54, 100. 